Groups Geom. Dyn. 5 (2011), 729-765

DOI $10.4171 / \mathrm{GGD} / 146$
Groups, Geometry, and Dynamics

(C) European Mathematical Society

\title{
Asphericity and small cancellation theory for rotation families of groups
}

\author{
Rémi Coulon
}

\begin{abstract}
Using small cancellation for rotation families of groups, we construct new examples of aspherical polyhedra.
\end{abstract}

Mathematics Subject Classification (2010). 20F65, 20F67, 20F06, 14F35.

Keywords. Geometric group theory, hyperbolic groups, small cancellation theory, asphericity, asymptotic cones.

\section{Introduction}

The goal of this article is to produce new examples of aspherical polyhedra. The construction we have in mind is the following: let $P$ be an aspherical simplicial complex and $Q$ a subcomplex of $P$. We define $\bar{P}$ by attaching to $P$ a cone of base $Q$. We are looking for conditions under which $\bar{P}$ remains aspherical. This kind of situation was already studied by J.H. C. Whitehead in the 1940s. In [21] and [22], he studied the second homotopy group of a space $\bar{P}$, obtained by attaching 2 -cells to a cell complex $P$. He proved that $\pi_{2}(\bar{P})$ exactly describes the identities between the relators that define the projection $\pi_{1}(P) \rightarrow \pi_{1}(\bar{P})$. In this paper we try to attach higher-dimensional cells.

Our main example is the following. Let $\mathbb{H}_{n}(\mathbb{C})$ be the complex $n$-dimensional hyperbolic space. We consider $\mathrm{SO}(n, 1)$ as the stabilizer of the real hyperbolic space $\mathbb{H}_{n}(\mathbb{R})$ in $\mathbb{H}_{n}(\mathbb{C})$. Let $G \subset \mathrm{SU}(n, 1)$ be a real lattice, i.e., a lattice of $\mathrm{SU}(n, 1)$ such that $H=G \cap \operatorname{SO}(n, 1)$ is still a lattice of $\operatorname{SO}(n, 1)$. We want to study the quotient $G /\langle\langle H\rangle\rangle$, where $\langle\langle H\rangle\rangle$ is the normal subgroup of $G$ generated by $H$.

Theorem. There exists a finite index subgroup $G^{\prime}$ of $G$ with the following property. Let $H^{\prime}$ be the group $G^{\prime} \cap \mathrm{SO}(n, 1)$. Let $\bar{P}$ be the space obtained by attaching to $\mathbb{H}_{n}(\mathbb{C}) / G^{\prime}$ a cone of base $\mathbb{H}_{n}(\mathbb{R}) / H^{\prime}$. The complex $\bar{P}$ is a classifying space for the group $\bar{G}^{\prime}=G^{\prime} /\left\langle\left\langle H^{\prime}\right\rangle\right\rangle$. 
This situation is similar to a result of N. Bergeron in [3] who proved that the map from the homology associated with $\mathbb{H}_{n}(\mathbb{R}) / G \cap \mathrm{SO}(n, 1)$ to the one of $\mathbb{H}_{n}(\mathbb{C}) / G$ is one to one. We also establish some analogue statements to our theorem for the following pairs (SO $(n, 1), \mathrm{SO}(k, 1)),(\mathrm{SU}(n, 1), \mathrm{SU}(k, 1))$ and $(S p(n, 1), S p(k, 1))$.

Our strategy is as follows. We endow the space $\bar{P}$ with a local hyperbolic geometry and apply a version of the Cartan-Hadamard Theorem. This will prove that the universal cover $\bar{X}$ of $\bar{P}$ is globally hyperbolic. We then deduce the asphericity from a kind of Rips' theorem (cf. Theorem 1.3.4): if $\bar{X}$ is a hyperbolic simplicial complex, it is sufficient to prove that $\bar{X}$ is locally contractible. This last local assumption follows from the property of the developing map.

The hyperbolic structure on $\bar{P}$ is constructed as follows. Given a subcomplex $Q$ of $P$, we endow the cone of base $Q$ with a metric modelled on the hyperbolic disc. An argument of Berestovskii tells us that if $Q$ is CAT(1) then the cone is CAT(-1) (see [5]). In particular it is hyperbolic. The problem is then to find some conditions so that the complex $\bar{P}$ remains locally hyperbolic.

With this aim in mind, we explore an idea of M. Gromov (see [15]) to extend the small cancellation theory to a so-called "rotation family" of groups. If $X$ is a metric space and $G$ a group acting on $X$ by isometries, a rotation family is a pairwise distinct collection $\left(Y_{i}, H_{i}\right)_{i \in I}$ such that

- $H_{i}$ is a subgroup of $G$ stabilizing $Y_{i} \subset X$,

- there is an action of $G$ on $I$ compatible with the one on $X$ (i.e., $Y_{g i}=g Y_{i}$ and $H_{g i}=g H_{i} g^{-1}$ for all $g \in G$ and all $i \in I$ ).

In order to study such a family, we define two quantities that respectively play the role of the largest piece and the smallest relator in the usual small cancellation theory. The constant $\Delta$ measures the overlap between two $Y_{i}$ 's whereas $\rho$ is the minimal translation length of a non-trivial element that belongs to an $H_{i}$.

Given a rotation family, we define the cone-off over $X$, denoted by $\dot{X}$, by attaching to $X$ cones of base $Y_{i}$. We consider the space $\bar{P}=\dot{X} / G$ whose fundamental group is $\bar{G}=G /\left\langle\left\langle H_{i}\right\rangle\right\rangle$. The small cancellation provides us a framework where it is possible to endow $\bar{P}$ with a local hyperbolic geometry. Moreover, this theory recovers the usual small cancellation (see [17] or [19]) and the small cancellation with graphs as well (see [15] or [18]).

We describe the space $\bar{P}$ as an orbifold using two kind of charts: the cones and the cone-off. Section 2 is dedicated to the study of the geometry of the cones. Adapting an argument of Berestovskii, we prove that the cone over a hyperbolic space remains hyperbolic (cf. Theorem 2.3.2). Section 3 deals with the cone-off $\dot{X}$. In particular we prove the following fact. Under small cancellation assumptions, the cone-off over a hyperbolic space is still hyperbolic (cf. Theorem 3.5.2). We choose for this proof an asymptotical point of view that involves ultra-limits as in [10]. The goal of the main technical lemma is to switch the cone-off construction and the ultra-limit: given a sequence of metric spaces $X_{n}$, we prove (see Corollary 3.5.9) that there exists a local isometry between the cone-off over the ultra-limit of $X_{n}$ and the ultra-limit of $\dot{X}_{n}$. 
Section 4 mixes all the previous ingredients to obtain the following theorems.

Theorem (cf. Theorem 4.2.2). There exist two positive numbers $\delta_{0}$ and $\Delta_{0}$ satisfying the following property.

Let $X$ be a geodesic, simply connected, $\delta$-hyperbolic space and $G$ a group acting properly by isometries on $X$. Let $\left(Y_{i}, H_{i}\right)_{i \in I}$ be a rotation family such that each $Y_{i}$ is strongly-quasi-convex.

Let $N$ be the normal subgroup of $G$ generated by the $H_{i}$ 's and $\bar{G}$ the quotient group $G / N$. Assume also that

$$
\frac{\delta}{\rho} \leqslant \delta_{0} \quad \text { and } \quad \frac{\Delta}{\rho} \leqslant \Delta_{0} .
$$

Then the universal cover of $\bar{P}, \bar{X}$ is hyperbolic and $\bar{G}$ acts properly by isometries on $\bar{X}$.

Moreover if $G$ (resp. $H_{i}$ ) acts co-compactly on $X$ (resp. $Y_{i}$ ) and $I / G$ is finite, then $\bar{X} / \bar{G}$ is compact. In particular $\bar{G}$ is hyperbolic.

Theorem (cf. Theorem 4.3.7). Under the same hypotheses, if $X$ is a $n$-dimensional simplicial complex such that every closed ball of $X$ and of each $Y_{i}$ is contractible in its appropriate neighbourhood, then $\bar{X}$ is contractible.

Remark. In some cases, the space $\bar{P}=\bar{X} / \bar{G}$ may be endowed with a sharper geometry than the hyperbolic one. For instance, M. Gromov constructed in a similar way CAT $(-1)$ polyhedra in order to produce infinite torsion groups (see [12], Chap. 12). In [14] M. Gromov introduced the notion of CAT $(-1, \varepsilon)$ spaces, an $\varepsilon$-perturbated version of CAT $(-1)$-spaces. This provides another framework to study small cancellation constructions which is not asymptotic.

In Section 5, we explain how to construct examples of rotation families that satisfy the small cancellation assumptions. To that end, we use the geometry of lattices and a result of $\mathrm{N}$. Bergeron about the profinite topology of finitely generated linear groups [2]. This leads to these new examples of aspherical polyhedra.

Question. The small cancellation for rotating families provides a framework to study quotient groups which looks very similar to the usual small cancellation. However the groups obtained in this way may have very different properties. For instance, a usual $C^{\prime}\left(\frac{1}{6}\right)$ small cancellation group acts properly discontinuously and co-compactly on a CAT(0)-cubical complex (see [23], Th. 1.2). In particular it cannot have Kazhdan's property (T) unless it is finite and cyclic. On the other hand, M. Gromov used in [15] (see also [1]) the small cancellation theory with graphs in order to construct Kazhdan groups. To that end he embedded expanding graphs in the Cayley graph of the group. We wonder if it is possible to construct other examples of Kazhdan groups using the small cancellation theory with rotation families. In particular we are interested in the following example. As above, let $G$ be a real lattice of $\operatorname{SU}(n, 1)$ and $H$ the subgroup $G \cap \operatorname{SO}(n, 1)$. Does $\bar{G}=G /\langle\langle H\rangle\rangle$ have the Kazhdan property $(\mathrm{T})$ ? 
Acknowledgements. I am grateful to Thomas Delzant for his invaluable help and advice during this work. Many thanks go to Nicolas Bergeron who explained me how to use the profinite topology. I thank also the referee for many useful comments and corrections.

\section{Hyperbolic spaces}

Let $X$ be a metric space. If $x$ and $x^{\prime}$ are two points of $X$, we denote by $\left|x^{\prime}-x\right|_{X}$ (or simply $\left|x^{\prime}-x\right|$ ) the distance between them. Although it may not be unique, we denote by $\left[x, x^{\prime}\right]$ a geodesic joining $x$ and $x^{\prime}$. Given a base point $x$, the Gromov product of two points $y$ and $z$ is defined by

$$
\langle y, z\rangle_{x}=\frac{1}{2}(|y-x|+|z-x|-|z-y|) .
$$

Let $\delta$ be a non-negative number. The space $X$ is $\delta$-hyperbolic if for all $x, y, z, t \in X$ we have $\langle x, z\rangle_{t} \geqslant \min \left\{\langle x, y\rangle_{t},\langle y, z\rangle_{t}\right\}-\delta$. $\mathbb{R}$-trees are very special examples of hyperbolic spaces.

Proposition-Definition 1.0.1 ([12], Chap. 2., Prop. 6, or [6], Chap. 3, Th. 4.1). An $\mathbb{R}$-tree is a geodesic space such that every two points are connected by a unique topological arc. A metric space is an $\mathbb{R}$-tree if and only if it is geodesic and 0hyperbolic.

Definition 1.0.2 (Quasi-isometry). Let $\eta$ be a non-negative number. A $(1, \eta)$-quasiisometry is a map $f: X \rightarrow Y$ between two metric spaces such that for all $x, x^{\prime} \in X$, we have

$$
\left|x^{\prime}-x\right|-\eta \leqslant\left|f\left(x^{\prime}\right)-f(x)\right| \leqslant\left|x^{\prime}-x\right|+\eta .
$$

The next result is a very easy case of the stability of quasi-geodesics. An asymptotic proof of this fact for a general $(\lambda, k)$-quasi-isometry can be found in [7].

Proposition 1.0.3. Let $\delta$ be a non-negative number. For all $\delta^{\prime}>\delta$, there exists $\eta>0$ satisfying the following property. Let $X$ be a metric space and $Y$ a $\delta$-hyperbolic space. If there exists a $(1, \eta)$-quasi-isometry from $X$ to $Y$, then $X$ is $\delta^{\prime}$-hyperbolic.

Proof. Let $f: X \rightarrow Y$ be a $(1, \eta)$-quasi-isometry. For all $x, y, z \in X$ we have

$$
\langle f(x), f(y)\rangle_{f(z)}-\frac{3}{2} \eta \leqslant\langle x, y\rangle_{z} \leqslant\langle f(x), f(y)\rangle_{f(z)}+\frac{3}{2} \eta .
$$

It follows that for all $x, y, z, t \in X$,

$$
\begin{aligned}
\langle x, z\rangle_{t} & \geqslant\langle f(x), f(z)\rangle_{f(t)}-\frac{3}{2} \eta \\
& \geqslant \min \left\{\langle f(x), f(y)\rangle_{f(t)},\langle f(y), f(z)\rangle_{f(t)}\right\}-\delta-\frac{3}{2} \eta \\
& \geqslant \min \left\{\langle x, y\rangle_{t},\langle y, z\rangle_{t}\right\}-(\delta+3 \eta) .
\end{aligned}
$$

Hence $X$ is $(\delta+3 \eta)$-hyperbolic. 
1.1. Ultra-limits of hyperbolic spaces. Let us recall the definition of the ultralimit of a sequence of pointed metric spaces and its link with hyperbolicity. For more details about this point of view see [9], [10] or [11].

A non-principal ultra-filter is a finite additive map $\omega: \mathcal{P}(\mathbb{N}) \rightarrow\{0,1\}$ that vanishes on every finite subset of $\mathbb{N}$ and is such that $\omega(\mathbb{N})=1$. A property $\mathcal{P}_{n}$ is true $\omega$-almost surely $\left(\omega\right.$-as) if $\omega\left(\left\{n \in \mathbb{N} / \mathcal{P}_{n}\right.\right.$ is true $\left.\}\right)=1$. A real sequence $\left(u_{n}\right)$ is $\omega$ essentially bounded ( $\omega$-eb) if there exists $M \in \mathbb{R}$ such that $\left|u_{n}\right| \leqslant M \omega$-as. If $l$ is a real number, we say that the $\omega$-limit of $\left(u_{n}\right)$ is $l$ and write $\lim _{\omega} u_{n}=l$ if $\left|l-u_{n}\right| \leqslant \varepsilon$ $\omega$-as for all $\varepsilon>0$. In particular, any $\omega$-eb sequence admits an $\omega$-limit (cf. [4]).

Let $\left(X_{n}, x_{n}^{0}\right)$ be a sequence of pointed metric spaces. We consider

$$
\prod_{\omega} X_{n}=\left\{\left(x_{n}\right) \mid x_{n} \in X_{n} \text { for all } n \in \mathbb{N} \text { and }\left(\left|x_{n}-x_{n}^{0}\right|\right) \text { is } \omega \text {-eb }\right\} .
$$

We endow this space with a pseudo-metric defined as follows:

$$
\left|\left(y_{n}\right)-\left(x_{n}\right)\right|=\lim _{\omega}\left|y_{n}-x_{n}\right| .
$$

Definition 1.1.1 (Ultra-limit of metric spaces). Let $\left(X_{n}, x_{n}^{0}\right)$ be a sequence of pointed metric spaces and $\omega$ a non-principal ultra-filter. The $\omega$-limit of $\left(X_{n}, x_{n}^{0}\right)$, denoted by $\lim _{\omega}\left(X_{n}, x_{n}^{0}\right)$ (or simply $\lim _{\omega} X_{n}$ ), is the quotient of $\prod_{\omega} X_{n}$ by the equivalence relation which identifies the points at distance zero.

The pseudo-distance on $\prod_{\omega} X_{n}$ induces a distance on $\lim _{\omega} X_{n}$.

Notation. (i) If $\left(x_{n}\right)$ is an element of $\prod_{\omega} X_{n}$, its image in $\lim _{\omega} X_{n}$ is denoted by $\lim _{\omega} x_{n}$.

(ii) For all $n \in \mathbb{N}$, let $Y_{n}$ be a subset of $X_{n}$. The set $\lim _{\omega} Y_{n}$ is defined by

$$
\lim _{\omega} Y_{n}=\left\{\lim _{\omega} y_{n} \mid\left(\left|y_{n}-x_{n}^{0}\right|\right) \text { is } \omega \text {-eb and } y_{n} \in Y_{n} \omega \text {-as }\right\} .
$$

Proposition 1.1.2. Let $\omega$ be a non-principal ultra-filter. Let $\left(\delta_{n}\right)$ be a sequence of non-negative numbers which admits an $\omega$-limit $\delta$. Let $\left(X_{n}, x_{n}^{0}\right)$ be a sequence of pointed metric spaces. If for all $n \in \mathbb{N}, X_{n}$ is $\delta_{n}$-hyperbolic, then the limit space $\lim _{\omega} X_{n}$ is $\delta$-hyperbolic.

Proof. Let $x=\lim _{\omega} x_{n}, y=\lim _{\omega} y_{n}, z=\lim _{\omega} z_{n}$ and $t=\lim _{\omega} t_{n}$ be four points of $\lim _{\omega} X_{n}$. Since $X_{n}$ is $\delta_{n}$-hyperbolic, we have, for all $n \in \mathbb{N},\left\langle x_{n}, z_{n}\right\rangle_{t_{n}} \geqslant$ $\min \left\{\left\langle x_{n}, y_{n}\right\rangle_{t_{n}},\left\langle y_{n}, z_{n}\right\rangle_{t_{n}}\right\}-\delta_{n}$. By taking the $\omega$-limit, it follows that $\langle x, z\rangle_{t} \geqslant$ $\min \left\{\langle x, y\rangle_{t},\langle y, z\rangle_{t}\right\}-\delta$. Thus $\lim _{\omega} X_{n}$ is $\delta$-hyperbolic.

Corollary 1.1.3. Let $\omega$ be a non-principal ultra-filter and $\left(\delta_{n}\right)$ a sequence of nonnegative numbers such that $\lim _{\omega} \delta_{n}=0$. Let $\left(X_{n}, x_{n}^{0}\right)$ be a sequence of pointed geodesic spaces. If $X_{n}$ is $\delta_{n}$-hyperbolic for all $n \in \mathbb{N}$, then the limit space $\lim _{\omega} X_{n}$ is an $\mathbb{R}$-tree. 
Proof. The $\omega$-limit of a sequence of geodesic spaces is still geodesic (cf. [20]). It follows that $\lim _{\omega} X_{n}$ is a geodesic, 0-hyperbolic metric space. Hence $\lim _{\omega} X_{n}$ is an $\mathbb{R}$-tree.

Proposition 1.1.4. Let $\omega$ be a non-principal ultra-filter and $\delta$ a non-negative number. Let $\left(X_{n}, x_{n}^{0}\right)$ be a sequence of pointed metric spaces whose diameters are bounded. If $\lim _{\omega} X_{n}$ is $\delta$-hyperbolic, then $X_{n}$ is $\delta^{\prime}$-hyperbolic $\omega$-as for all $\delta^{\prime}>\delta$. In particular there exists $n \in \mathbb{N}$ such that $X_{n}$ is $\delta^{\prime}$-hyperbolic.

Proof. Assume that the proposition is false. Then $\lim _{\omega} X_{n}$ is $\delta$-hyperbolic and nevertheless there exists $\delta^{\prime}>\delta$ such that $X_{n}$ is $\omega$-as not $\delta^{\prime}$-hyperbolic. Thus we can find four sequences $\left(x_{n}\right),\left(y_{n}\right),\left(z_{n}\right)$ and $\left(t_{n}\right)$ satisfying the following properties:

(i) $x_{n}, y_{n}, z_{n}, t_{n} \in X_{n}$ for all $n \in \mathbb{N}$,

(ii) $\left\langle x_{n}, z_{n}\right\rangle_{t_{n}}<\min \left\{\left\langle x_{n}, y_{n}\right\rangle_{t_{n}},\left\langle y_{n}, z_{n}\right\rangle_{t_{n}}\right\}-\delta^{\prime} \omega$-as.

Since $\left(\operatorname{diam}\left(X_{n}\right)\right)$ is bounded, these four sequences define four points of $\lim _{\omega} X_{n}$, respectively $x, y, z$ and $t$. After taking the $\omega$-limit in the previous inequality we obtain

$$
\langle x, z\rangle_{t} \leqslant \min \left\{\langle x, y\rangle_{t},\langle y, z\rangle_{t}\right\}-\delta^{\prime}<\min \left\{\langle x, y\rangle_{t},\langle y, z\rangle_{t}\right\}-\delta .
$$

Hence $\lim _{\omega} X_{n}$ is not $\delta$-hyperbolic. Contradiction.

1.2. Quasi-convexity. If $X$ is a geodesic space, there is another way to characterize the hyperbolicity using geodesic triangles. Let $\delta$ be a non-negative number. A geodesic triangle is $\delta$-thin if each one of its sides is contained in the $\delta$-neighbourhood of the union of the two others.

Proposition 1.2.1 (cf. [6], Chap. 1, Prop. 3.6, or [12], Chap. 3 §2). Let $\delta$ be a nonnegative number. Consider a geodesic space $X$.

(i) If $X$ is $\delta$-hyperbolic, then every geodesic triangle of $X$ is $4 \delta$-thin.

(ii) If every geodesic triangle of $X$ is $\delta$-thin, then $X$ is $8 \delta$-hyperbolic.

Corollary 1.2.2. Let $x, x^{\prime}, y$ and $y^{\prime}$ be four points of a geodesic, $\delta$-hyperbolic space $X$. If $u$ is a point of $\left[x, x^{\prime}\right]$ such that $|u-x|>|y-x|+8 \delta$ and $\left|u-x^{\prime}\right|>\left|y^{\prime}-x^{\prime}\right|+8 \delta$, then $u$ lies in the 8 $\delta$-neighbourhood of $\left[y, y^{\prime}\right]$.

Proof. Since the triangles $\left[x, y, y^{\prime}\right]$ and $\left[x, x^{\prime}, y^{\prime}\right]$ are $4 \delta$-thin, we can find a point $v$ in $[x, y] \cup\left[y, y^{\prime}\right] \cup\left[y^{\prime}, x^{\prime}\right]$ such that $|v-u| \leqslant 8 \delta$. We will show that $v \in\left[y, y^{\prime}\right]$. Suppose that $v \in[x, y]$ (the case $v \in\left[x^{\prime}, y^{\prime}\right]$ is symmetric). The triangle inequality gives

$$
|u-x| \leqslant|u-v|+|v-x| \leqslant|y-x|+8 \delta,
$$

a contradiction. Consequently, $u$ lies in the $8 \delta$-neighourhood of $\left[y, y^{\prime}\right]$. 
Definition 1.2.3 (Quasi-convexity). Let $\alpha$ be a non-negative number. A subset $Y$ of a geodesic metric space $X$ is $\alpha$-quasi-convex if every geodesic between two points of $Y$ is contained in the $\alpha$-neighbourhood of $Y$.

Notation. We denote by $Y^{+\alpha}$ the $\alpha$-neighbourhood of $Y$.

Proposition 1.2.4 (Cf. [8], Lemma 2.2.2). Let $\delta, \alpha \geqslant 0$. Let $X$ be a geodesic, $\delta$-hyperbolic space. If $Y$ and $Z$ are two $\alpha$-quasi-convex subsets of $X$, then we have

$$
\operatorname{diam}\left(Y^{+A} \cap Z^{+A}\right) \leqslant \operatorname{diam}\left(Y^{+\alpha+10 \delta} \cap Z^{+\alpha+10 \delta}\right)+2 A+20 \delta
$$

for all $A \geqslant 0$.

Proof. Let $x$ and $x^{\prime}$ be two points of $Y^{+A} \cap Z^{+A}$ such that $\left|x^{\prime}-x\right| \geqslant 2 A+20 \delta$. We choose

(i) two points $t$ and $t^{\prime}$ of $\left[x, x^{\prime}\right]$ such that $|t-x|=\left|t^{\prime}-x^{\prime}\right|=A+10 \delta$,

(ii) two points $y$ and $y^{\prime}$ of $Y$ such that $|y-x|,\left|y^{\prime}-x^{\prime}\right| \leqslant A+\delta$.

Applying Corollary 1.2.2, $t$ belongs to the $8 \delta$-neighbourhood of $\left[y, y^{\prime}\right]$. Since $Y$ is $\alpha$ quasi-convex, $\left[y, y^{\prime}\right]$ lies in the $\alpha$-neighbourhood of $Y$. Hence $t$ belongs to $Y^{+\alpha+10 \delta}$. We prove in the same way that $t$ belongs to $Z^{+\alpha+10 \delta}$. The same fact holds for $t^{\prime}$. Thus

$$
\left|x^{\prime}-x\right|-2 A-20 \delta=\left|t^{\prime}-t\right| \leqslant \operatorname{diam}\left(Y^{+\alpha+10 \delta} \cap Z^{+\alpha+10 \delta}\right) .
$$

The above inequality is true for all $x, x^{\prime} \in Y^{+A} \cap Z^{+A}$, hence

$$
\operatorname{diam}\left(Y^{+A} \cap Z^{+A}\right) \leqslant \operatorname{diam}\left(Y^{+\alpha+10 \delta} \cap Z^{+\alpha+10 \delta}\right)+2 A+20 \delta .
$$

Corollary 1.2.5. Let $\omega$ be a non-principal ultra-filter and $\left(\delta_{n}\right)$ a sequence of real numbers such that $\lim _{\omega} \delta_{n}=0$. For all $n \in \mathbb{N}$, let $\left(X_{n}, x_{n}^{0}\right)$ be a pointed, geodesic, $\delta_{n}$-hyperbolic space and let $Y_{n}, Z_{n}$ be two $10 \delta_{n}$-quasi-convex subsets of $X_{n}$. Let $X=\lim _{\omega}\left(X_{n}, x_{n}^{0}\right)$ and $Y=\lim _{\omega} Y_{n}, Z=\lim _{\omega} Z_{n}$. We have

$$
\operatorname{diam}(Y \cap Z) \leqslant \lim _{\omega} \operatorname{diam}\left(Y_{n}^{+20 \delta_{n}} \cap Z_{n}^{+20 \delta_{n}}\right) .
$$

Proof. Let $x$ and $x^{\prime}$ be two points of $Y \cap Z$. Since $x, x^{\prime} \in Y$, we can find two sequences $\left(x_{n}\right)$ and $\left(x_{n}^{\prime}\right)$ such that $x=\lim _{\omega} x_{n}, x^{\prime}=\lim _{\omega} x_{n}^{\prime}$ and $x_{n}, x_{n}^{\prime} \in Y_{n} \omega$-as. Moreover, $x$ and $x^{\prime}$ belong to $Z$, thus if $A>0$ is given, $x_{n}$ and $x_{n}^{\prime}$ belong to $Z_{n}^{+A}$ $\omega$-as. Using Proposition 1.2.4, we have

$$
\left|x_{n}^{\prime}-x_{n}\right| \leqslant \operatorname{diam}\left(Y_{n}^{+A} \cap Z_{n}^{+A}\right) \leqslant \operatorname{diam}\left(Y_{n}^{+20 \delta_{n}} \cap Z_{n}^{+20 \delta_{n}}\right)+2 A+20 \delta_{n} .
$$

By taking the $\omega$-limit, we obtain

$$
\left|x^{\prime}-x\right| \leqslant \lim _{\omega} \operatorname{diam}\left(Y_{n}^{+20 \delta_{n}} \cap Z_{n}^{+20 \delta_{n}}\right)+2 A .
$$


This inequality is true for all $A>0$ and $x, x^{\prime} \in Y \cap Z$, thus

$$
\operatorname{diam}(Y \cap Z) \leqslant \lim _{\omega} \operatorname{diam}\left(Y_{n}^{+20 \delta_{n}} \cap Z_{n}^{+20 \delta_{n}}\right) .
$$

We need in Section 3 a little stronger condition than quasi-convexity.

Definition 1.2.6. Let $X$ be a $\delta$-hyperbolic space. A subset $Y$ of $X$ is strongly quasiconvex if for all $x, x^{\prime} \in Y$ there exist $p, p^{\prime} \in Y$ such that $|x-p| \leqslant 10 \delta,\left|x^{\prime}-p^{\prime}\right| \leqslant$ $10 \delta$ and the path $[x, p] \cup\left[p, p^{\prime}\right] \cup\left[p^{\prime}, x^{\prime}\right]$ lies in $Y$.

Remark. Since any geodesic triangle of $X$ is $4 \delta$-thin, any strongly quasi-convex space is $10 \delta$-quasi-convex. Given a $10 \delta$-quasi-convex subset $Y$ of $X$, there is a way to find a subset of $X$, a little larger than $Y$, that is strongly quasi-convex. To that end, we define the cylinder of a subset.

Definition 1.2.7. Let $Y$ be a subset of a geodesic $\delta$-hyperbolic space $X$. The cylinder of $Y$, denoted by $\operatorname{cyl}(Y)$, is the set of all points which are in the $10 \delta$-neighbourhood of a geodesic of $X$ joining two points of $Y$.

Lemma 1.2.8. Let $Y$ be a $10 \delta$-quasi-convex subset of a geodesic, $\delta$-hyperbolic space $X$. The set cyl $(Y)$ is contained in $Y^{+20 \delta}$ and is strongly quasi-convex.

Proof. By definition of quasi-convexity, any geodesic joining two points of $Y$ lies in $Y^{+10 \delta}$. It follows that $\operatorname{cyl}(Y) \subset Y^{+20 \delta}$. Let $x$ and $x^{\prime}$ be two points of $\operatorname{cyl}(Y)$. By definition there exist two points of $Y, y_{1}$ and $y_{2}$ (resp. $y_{1}^{\prime}$ and $y_{2}^{\prime}$ ) such that $x$ (resp. $x^{\prime}$ ) belongs to the $10 \delta$-neighbourhood of $\left[y_{1}, y_{2}\right]$ (resp. $\left.\left[y_{1}^{\prime}, y_{2}^{\prime}\right]\right)$. We denote $p$ and $p^{\prime}$ the respective projections of $x$ and $x^{\prime}$ on $\left[y_{1}, y_{2}\right]$ and $\left[y_{1}^{\prime}, y_{2}^{\prime}\right]$.

By construction, the geodesic segments $[x, p]$ and $\left[p^{\prime}, x^{\prime}\right]$ are contained in $\operatorname{cyl}(Y)$ and shorter than $10 \delta$.

Since the triangles $\left[y_{2}, p, p^{\prime}\right]$ and $\left[y_{2}, y_{2}^{\prime}, p^{\prime}\right]$ are $4 \delta$-thin, $\left[p, p^{\prime}\right]$ stays in the $8 \delta$ neighbourhood of $\left[p, y_{2}\right] \cup\left[y_{2}, y_{2}^{\prime}\right] \cup\left[y_{2}^{\prime}, p^{\prime}\right]$. However these segments are parts of geodesics between two points of $Y$. Thus $\left[p, p^{\prime}\right]$ lies in $\operatorname{cyl}(Y)$.

Hence $[x, p] \cup\left[p, p^{\prime}\right] \cup\left[p^{\prime}, x^{\prime}\right]$ lies in $\operatorname{cyl}(Y)$.

\subsection{Asphericity}

Notation. If $X$ is a simplicial complex, we denote by $X^{(k)}$ its $k$-skeleton.

In this part we prove a version of the famous Rips' theorem: a hyperbolic simplicial complex which is locally aspherical is globally aspherical. Let $X$ be a metric space and $d$ a positive number. The Rips' polyhedron of $X$ denoted by $P_{d}(X)$ is a simplicial complex defined as follows. The simplices of $P_{d}(X)$ are the finite subsets of $X$ of diameter less than $d$. It is known (see [13], Section 2.2) that if $X$ is geodesic $\delta$ hyperbolic, then for all $d \geqslant 4 \delta$ the polyhedron $P_{d}(X)$ is contractible. More precisely, we have the following proposition. 
Proposition 1.3.1 (cf. [6], Chap. 5, Prop. 1.1). Let $X$ be a geodesic, $\delta$-hyperbolic space. Let $d \geqslant 4 \delta$ and $n \in \mathbb{N}$. The polyhedron $P_{d}^{(n+1)}(X)$ is $n$-connected.

Before studying the case of an arbitrary simplicial complex, we prove the following proposition.

Proposition 1.3.2. Let $X$ be a n-dimensional simplicial complex. Let $d>1$. Assume that for all $r \leqslant 2(n+1) d$ and for all $x \in X$ the closed ball $\bar{B}(x, r)$ is contractible in $B(x, r+d)$. Then there exist two maps: $f: X \rightarrow P_{d}^{(n+1)}\left(X^{(0)}\right)$ and $g: P_{d}^{(n+1)}\left(X^{(0)}\right) \rightarrow X$ such that $g \circ f$ is homotopic to $\operatorname{id}_{X}$.

Proof. In this proof we denote by $P$ the $(n+1)$-skeleton of the Rips' polyhedron $P_{d}\left(X^{(0)}\right)$. We define $f: X^{(0)} \rightarrow P$ by $f(x)=\{x\}$. Let $k \leqslant n$. If $\sigma$ is a $k$-simplex of $X$, its diameter is less than $d$. Thus the set of its vertices defines a $k$-simplex of $P$. Hence $f$ induces a simplicial map from $X$ to $P$. We now define by induction a map $g: P \rightarrow X$.

First we define a map $g^{(0)}: P^{(0)} \rightarrow X$ by $g^{(0)}(\{x\})=x$. Assume now that we have already defined a continuous map $g^{(k)}: P^{(k)} \rightarrow X$ with the following property: for each $l$-simplex $\sigma$ of $P^{(k)}$, for every vertex $x$ of $\sigma, g^{(k)}(\sigma)$ is contained in $B\left(g^{(k)}(x), 2 l d\right)$. Let $\sigma$ be a $(k+1)$-simplex of $P^{(k+1)}$ whose faces are $\sigma_{0}, \ldots, \sigma_{k+1}$. Choose a vertex $x$ of $\sigma$. The function $g^{(k)}$ maps the border $\partial \sigma=\bigcup_{i=0}^{k+1} \sigma_{i}$ onto a $k$ sphere of $X$ contained in $B\left(g^{(k)}(x),(2 k+1) d\right)$. However this sphere is contractible in $B\left(g^{(k)}(x), 2(k+1) d\right)$. We define $g^{(k+1)}(\sigma)$ by choosing a homotopy which contracts $g^{(k)}(\partial \sigma)$ to a point. This defines a continuous map $g^{(k+1)}: P^{(k+1)} \rightarrow X$ which coincides with $g^{(k)}$ on $P^{(k)}$ and satisfies the following property: $g^{(k)}(\sigma)$ is contained in $B\left(g^{(k)}(x), 2 l d\right)$ for all $l$-simplex $\sigma$ of $P^{(k+1)}$ and for every vertex $x$ of $\sigma$. We choose for $g$ the map $g^{(n+1)}$.

Lemma 1.3.3. For all $k \leqslant n+1$ there is a continuous map $H^{(k)}: X^{(k)} \times[0,1] \rightarrow X$ satisfying the following properties:

(i) $\left.H^{(k)}\right|_{X^{(k)} \times\{0\}}=\mathrm{id}_{X^{(k)}}$ and $\left.H^{(k)}\right|_{X^{(k)} \times\{1\}}=\left.g \circ f\right|_{X^{(k)}}$.

(ii) $H^{(k)}(\sigma \times[0,1])$ is contained in $B(x,(2 l+1) d)$ for each $l$-simplex $\sigma$ of $X^{(k)}$ and for every vertex $x$ of $\sigma$.

Proof. We prove this result by induction on $k$. The restriction of $g \circ f$ to $X^{(0)}$ is the identity, thus the proposition is obvious for the 0 -skeleton. Assume now that the lemma is true for $k \leqslant n$. Consider a $(k+1)$-simplex $\sigma$ of $X^{(k+1)}$. We choose a vertex $x$ of $\sigma$. By definition of $g$ the set $g \circ f(\sigma)$ is contained in $B(x,(2 k+2) d)$. Moreover, the induction assumption gives that $H^{(k)}(\partial \sigma \times[0,1]) \subset B(x,(2 k+2) d)$. Thus the subset $\sigma \cup H^{(k)}(\partial \sigma \times[0,1]) \cup g \circ f(\sigma)$ is a $(k+1)$-sphere of $X$ contained in $B(x,(2 k+2) d)$. This sphere is therefore contractible in $B(x,(2 k+3) d)$. By choosing a homotopy that contracts it to a point, we define a map $H^{(k+1)}: \sigma \times[0,1] \rightarrow$ $X$ such that 
(i) $\left.H^{(k+1)}\right|_{\sigma \times\{0\}}=\operatorname{id}_{\sigma}$ and $\left.H^{(k+1)}\right|_{\sigma \times\{1\}}=\left.g \circ f\right|_{\sigma}$,

(ii) $\left.H^{(k+1)}\right|_{\partial \sigma \times[0,1]}=\left.H^{(k)}\right|_{\partial \sigma \times[0,1]}$,

(iii) $H^{(k+1)}(\sigma \times[0,1]) \subset B(x,(2 k+3) d)$.

This defines a map $H^{(k+1)}: X^{(k+1)} \times[0,1] \rightarrow X$ which satisfies the properties of the lemma.

End of the proof of Proposition 1.3.2. The map $H^{(n+1)}: X \times[0,1] \rightarrow X$ is a homotopy between $g \circ f$ and $\operatorname{id}_{X}$.

Theorem 1.3.4. Let $X$ be a $\delta$-hyperbolic, $n$-dimensional, simplicial complex. Assume that the ball $B(x, r)$ is homotopic to zero in $B(x, r+4 \delta)$ for all $r \leqslant 8(n+1) \delta$ and all $x \in X$. Then all homotopy groups of $X$ are trivial. Hence $X$ is contractible.

Proof. We fix $d=4 \delta$. From Proposition 1.3.1 it follows that the Rips polyhedron $P=P_{d}^{(n+1)}\left(X^{(0)}\right)$ is $n$-connected. Moreover, the fact that the small balls are contractible gives two maps $f: X \rightarrow P$ and $g: P \rightarrow X$ such that $g \circ f$ is homotopic to id $X$. It follows that $X$ is $n$-connected as well. Since $X$ is $n$-dimensional, all the higher homotopy groups of $X$ are trivial by Hurewicz's theorem.

\section{Cone over a metric space}

In this section we prove an asymptotic version of the Berestovskii's theorem concerning the hyperbolicity of a cone with a locally hyperbolic base. From now on, we fix a positive number $r_{0}$ whose value will be made precise in Section 4.

\subsection{Definition}

Definition 2.1.1. Let $Y$ be a metric space. The cone over $Y$, denoted by $C(Y)$ is the quotient of $Y \times\left[0, r_{0}\right]$ by the equivalence relation defined as follows. Two points $\left(y_{1}, r_{1}\right)$ and $\left(y_{2}, r_{2}\right)$ are equivalent if $r_{1}=r_{2}=0$ or $\left(y_{1}, r_{1}\right)=\left(y_{2}, r_{2}\right)$.

Notation. The equivalence class of $(y, 0)$, called the vertex of the cone, is denoted $v_{Y}$ (or simply $v$ ). The equivalence class of any other point $(y, r)$ is still denoted by $(y, r)$.

Hyperbolic metric on a cone. We define a metric on $C(Y)$ as $\mathrm{M}$. Bridson and A. Haefliger do in [5], Chap. I.5. If $y$ and $y^{\prime}$ are two points of $Y$, we consider the angle $\theta\left(y, y^{\prime}\right)$ defined by $\theta\left(y, y^{\prime}\right)=\min \left\{\pi, \frac{\left|y^{\prime}-y\right|}{\sinh r_{0}}\right\}$.

Proposition 2.1.2 ([5], Chap. I.5, Prop. 5.9). The following formula defines a distance on the cone $C\left(Y, r_{0}\right)$.

$$
\left|\left(y^{\prime}, r^{\prime}\right)-(y, r)\right|=\operatorname{arccosh}\left(\cosh r \cosh r^{\prime}-\sinh r \sinh r^{\prime} \cos \theta\left(y, y^{\prime}\right)\right) .
$$


Remarks. The distance on the cone has the following interpretation. Given two points $(y, r)$ and $\left(y^{\prime}, r^{\prime}\right)$ of $C(Y)$, the distance between them is the distance between two points of the hyperbolic disc respectively distant from the centre of $r$ and $r^{\prime}$, such that the central angle between them is $\theta\left(y, y^{\prime}\right)$.

It is important to notice that $\left|\left(y^{\prime}, r^{\prime}\right)-(y, r)\right|$ is a continuous function of $y, y^{\prime}, r$ and $r^{\prime}$.

The cone $C(Y)$ is the ball of centre $v$ and of radius $r_{0}$ of the space $C_{-1}\left(\frac{Y}{\sinh r_{0}}\right)$ defined in [5], Chap. I.5.

Proposition 2.1.3 ([5], Chap. I.5, Prop. 5.10). Let $(y, r)$ and $\left(y^{\prime}, r^{\prime}\right)$ be two points of $C\left(Y, r_{0}\right)$.

(i) If $r, r^{\prime}>0$ and $\theta\left(y, y^{\prime}\right)<\pi$, then there is a bijection between the set of geodesics joining $y$ and $y^{\prime}$ in $Y$ and the set of geodesics joining $(y, r)$ and $\left(y^{\prime}, r^{\prime}\right)$ in $C(Y)$.

(ii) In all other cases, there is a unique geodesic joining $(y, r)$ and $\left(y^{\prime}, r^{\prime}\right)$.

Examples. (i) If $Y$ is a circle, endowed with its length metric, whose perimeter is $2 \pi \sinh r_{0}$, then the cone $C(Y)$ is the hyperbolic disc of radius $r_{0}$.

(ii) If $Y$ is isometric to a line, then $C(Y) \backslash\{v\}$ is the universal cover of the punctured hyperbolic disc of radius $r_{0}$.

Relation between the cone and its base. In order to compare the cone $C(Y)$ and its base $Y$ we consider two maps:

$$
\begin{aligned}
\iota: Y & \rightarrow C(Y), & p: C(Y) \backslash\{v\} & \rightarrow Y, \\
y & \mapsto\left(y, r_{0}\right), & (y, r) & \mapsto y .
\end{aligned}
$$

Proposition 2.1.4. Let $(y, r)$ and $\left(y^{\prime}, r^{\prime}\right)$ be two points of $C(Y)$. Then

$$
2 \min \left\{r, r^{\prime}\right\} \frac{\theta\left(y, y^{\prime}\right)}{\pi} \leqslant\left|\left(y^{\prime}, r^{\prime}\right)-(y, r)\right| \leqslant\left|r^{\prime}-r\right|+\sqrt{\sinh r \sinh r^{\prime}} \theta\left(y, y^{\prime}\right) .
$$

In particular, let $x$ be a point of $C(Y)$ whose distance to $v$ is at least $\frac{r_{0}}{2}$. Then for every point $x^{\prime}$ in the ball $B\left(x, \frac{r_{0}}{3}\right)$ we have $\left|p\left(x^{\prime}\right)-p(x)\right|_{Y} \leqslant \frac{3 \pi \sinh r_{0}}{r_{0}}\left|x^{\prime}-x\right|_{C(Y)}$.

Proof. The inequalities follow from the facts that

- the map $t \rightarrow \operatorname{arccosh}(1+a(1-\cos t))$ is concave,

- for all $t \geqslant 0, \operatorname{arccosh}(a+t) \leqslant \operatorname{arccosh}(a)+\sqrt{2 t}$.

Consider now a point $x=(y, r)$ of $C(Y)$ whose distance to $v$ is at least $\frac{r_{0}}{2}$. If $x^{\prime}=\left(y^{\prime}, r^{\prime}\right)$ belongs to the ball $B\left(x, \frac{r_{0}}{3}\right)$, then $\left|x^{\prime}-x\right|<\frac{r_{0}}{3}<r<r+r^{\prime}$. It follows that $\theta\left(y, y^{\prime}\right)<\pi$. Moreover $r^{\prime} \geqslant \frac{r_{0}}{6}$. Using the previous inequality, we obtain $\frac{r_{0}}{3 \pi \sinh r_{0}}\left|y^{\prime}-y\right| \leqslant \frac{2 \min \left\{r, r^{\prime}\right\}}{\pi \sinh r_{0}}\left|y^{\prime}-y\right| \leqslant\left|x^{\prime}-x\right|$. 
Proposition 2.1.5. Let $\mu: \mathbb{R}^{+} \rightarrow \mathbb{R}^{+}$be the map defined by $\left|\iota\left(y^{\prime}\right)-\iota(y)\right|=$ $\mu\left(\left|y^{\prime}-y\right|\right)$ for all $y, y^{\prime} \in Y$. Then $\mu$ is a non-decreasing, continuous, concave map satisfying the following properties:

(i) $\mu\left(t+t^{\prime}\right) \leqslant \mu(t)+\mu\left(t^{\prime}\right)$ for all $t, t^{\prime} \geqslant 0$ (subadditivity),

(ii) $\frac{2 r_{0}}{\pi \sinh r_{0}} \min \left\{\pi \sinh r_{0}, t\right\} \leqslant \mu(t) \leqslant t$ for all $t \geqslant 0$.

Proof. By construction, the map $\mu$ is defined by

$$
\mu(t)=\operatorname{arccosh}\left(\cosh ^{2} r_{0}-\sinh ^{2} r_{0} \cos \left(\min \left\{\pi, \frac{t}{\sinh r_{0}}\right\}\right)\right) .
$$

The properties of $\mu$ follow from its concavity (cf. Fig. 1).

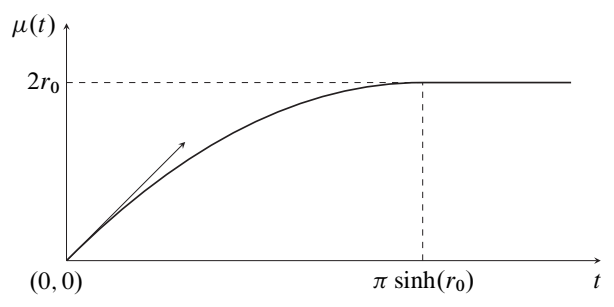

Figure 1. Graph of $\mu$.

\subsection{Cone and hyperbolicity}

Lemma 2.2.1. Let $\omega$ be a non-principal ultra-filter. Let $\left(Y_{n}, y_{n}^{0}\right)$ be a sequence of pointed metric spaces. We assume that the sequence $\left(\operatorname{diam}\left(Y_{n}\right)\right)$ is bounded. The spaces $C\left(\lim _{\omega}\left(Y_{n}, y_{n}^{0}\right)\right)$ and $\lim _{\omega}\left(C\left(Y_{n}\right), \iota_{n}\left(y_{n}^{0}\right)\right)$ are isometric.

Proof. Denote by $Y$ the $\operatorname{limit}_{\operatorname{space}} \lim _{\omega}\left(Y_{n}, y_{n}^{0}\right)$. We define a map $f: C(Y) \rightarrow$ $\lim _{\omega} C\left(Y_{n}\right)$ by $f\left(\lim _{\omega} y_{n}, r\right)=\lim _{\omega}\left(y_{n}, r\right)$. Since Formula (1), giving the distance in a cone, is continuous, the map $f$ preserves the distances. Consider now a point $x=\lim _{\omega}\left(y_{n}, r_{n}\right)$ of $\lim _{\omega} C\left(Y_{n}\right)$. By assumption, the sequences $\left(r_{n}\right)$ and $\left(\left|y_{n}-y_{n}^{0}\right|\right)$ are bounded. Thus we may consider the real number $r=\lim _{\omega} r_{n}$ and the point $y=\lim _{\omega} y_{n}$ of $Y$. Furthermore,

$$
|f(y, r)-x|=\lim _{\omega}\left|\left(y_{n}, r\right)-\left(y_{n}, r_{n}\right)\right|=\lim _{\omega}\left|r-r_{n}\right|=0 .
$$

It follows that $f(y, r)=x$. Hence $f$ is onto.

Lemma 2.2.2. Let $Y$ be a metric space. If every ball of radius $\pi \sinh r_{0}$ of $Y$ is an $\mathbb{R}$-tree, then the cone $C(Y)$ is $\mathrm{CAT}(-1)$. In particular this cone is $\ln 3$-hyperbolic.

Proof. Let $T$ be a geodesic triangle of $Y$ whose perimeter is smaller than $2 \pi \sinh r_{0}$. It is contained in a ball of radius $\pi \sinh r_{0}$. It follows that $T$ is 0 -thin. Consequently 
the rescaled space $\frac{Y}{\sinh r_{0}}$ is CAT(1) (cf. [5]). Using a Berestovskii's theorem (cf. [5], Chap. II.3, Th. 3.14), the cone $C(Y)$ is CAT(-1). In particular it is $\ln 3$-hyperbolic.

Proposition 2.2.3. Let $\varepsilon>0$. There exists $\delta>0$ satisfying the following property. Let $X$ be a geodesic space such that each ball of radius $\pi \sinh r_{0}$ of $X$ is $\delta$-hyperbolic. Let $Y$ be a $10 \delta$-quasi-convex subset of $X$. The cone $C(Y)$ is $(\ln 3+\varepsilon)$-hyperbolic.

Proof. Assume that the proposition is false. For all $n \in \mathbb{N}$, we can find

- a geodesic space $X_{n}$ whose balls of radius $\pi \sinh r_{0}$ are $\delta_{n}$-hyperbolic, with $\delta_{n}=o(1)$,

- a $10 \delta_{n}$-quasi-convex subset $Y_{n}$ of $X_{n}$,

such that the cone $C\left(Y_{n}\right)$ is not $(\ln 3+\varepsilon)$-hyperbolic. We denote by $\tilde{X}_{n}$ the space $X_{n}$ endowed with the metric

$$
\left|x^{\prime}-x\right|_{\tilde{X}_{n}}=\min \left\{\pi \sinh r_{0},\left|x^{\prime}-x\right|_{X_{n}}\right\}
$$

The set $Y_{n}$ viewed as a subspace of $\tilde{X}_{n}$ is denoted by $\tilde{Y}_{n}$. We choose a non-principal ultra-filter $\omega$, the limit space $\tilde{X}=\lim _{\omega} \tilde{X}_{n}$ and the subspace $\tilde{Y}=\lim _{\omega} \tilde{Y}_{n}$. Each ball of radius $\pi \sinh r_{0}$ of $\tilde{Y}_{n}$ is $\delta_{n}$-hyperbolic. Hence each ball of radius $\pi \sinh r_{0}$ of $\tilde{Y}$ is 0-hyperbolic. Moreover $Y_{n}$ is $10 \delta_{n}$-quasi-convex. It follows that, for all $y \in \tilde{Y}$, the set $\widetilde{Y} \cap B\left(y, \pi \sinh r_{0}\right)$ is an $\mathbb{R}$-tree. Using Lemma 2.2.2, $C(\tilde{Y})$ is $\ln$ 3-hyperbolic. Moreover, the diameter of $\widetilde{Y}_{n}$ is uniformly bounded. Hence Lemma 2.2.1 tells us that $C(\tilde{Y})$ and $\lim _{\omega} C\left(\tilde{Y}_{n}\right)$ are isometric. Thus there exists $n \in \mathbb{N}$ such that $C\left(\tilde{Y}_{n}\right)$ is $(\ln 3+\varepsilon)$-hyperbolic. However $C\left(Y_{n}\right)$ and $C\left(\tilde{Y}_{n}\right)$ are isometric. Contradiction.

\subsection{Group acting on a cone}

Definition 2.3.1. Let $X$ be a metric space and $G$ a group acting on $X$ by isometries. For all $g \in G$ the translation length of $g$, denoted by $[g]_{X}$ (or simply $[g]$ ) is

$$
[g]_{X}=\inf _{x \in X}|g x-x| .
$$

The injectivity radius of $G$ on $X$ is $r_{\mathrm{inj}}(G, X)=\inf _{g \in G \backslash\{1\}}[g]_{X}$.

Let $Y$ be a metric space. We fix a group $H$ acting by isometries on $Y$. We assume that this action is proper, that is for all $y \in Y$ there exists $r>0$ such that the set $\{h \in H \mid h \cdot B(y, r) \cap B(y, r) \neq \emptyset\}$ is finite. We denote by $\bar{Y}$ the quotient $Y / H$ and by $\bar{y}$ the image in $\bar{Y}$ of a point $y \in Y$. Since $H$ acts properly on $Y$, the quotient $\bar{Y}$ may be endowed with a metric defined by $\left|\overline{y^{\prime}}-\bar{y}\right|_{\bar{Y}}=\inf _{h \in H}\left|h y^{\prime}-y\right|_{Y}$.

We extend the action of $H$ to the cone $C(Y)$ by homogeneity: If $x=(y, r)$ is a point of $C(Y)$ and $h$ an element of $H$, then $h x$ is defined by $h x=(h y, r)$. The 
group $H$ acts by isometries on $C(Y)$. Note that if $Y$ is not compact, this action is no more proper. However the relation $\left|\bar{x}^{\prime}-\bar{x}\right|=\inf _{h \in H}\left|h x^{\prime}-x\right|_{C(Y)}$ still defines a metric on the quotient $C(Y) / H$. Moreover $C(Y / H)$ and $C(Y) / H$ are isometric.

Theorem 2.3.2 (First hyperbolicity theorem). Let $\varepsilon>0$. There exists $\delta>0$ satisfying the following property. Let $X$ be a $\delta$-hyperbolic, geodesic space and $Y$ a $10 \delta$-quasiconvex subset of $X$. Assume that $H$ is a group acting by isometries on $X$ such that $H$ stabilizes $Y$. If $r_{\mathrm{inj}}(H, Y)>2 \pi \sinh r_{0}$, then the space $C(Y) / H$ is $(\ln 3+\varepsilon)$ hyperbolic.

Proof. We consider the constant $\delta>0$ given by Proposition 2.2.3. Let $X$ be a $\delta$ hyperbolic, geodesic space and $Y$ a $10 \delta$-quasi-convex subset of $X$. Assume that $H$ is a group acting by isometries on $X$ such that $H$ stabilizes $Y$ and $r_{\text {inj }}(H, Y)>2 \pi \sinh r_{0}$. The spaces $X / H$ and $Y / H$ satisfy the assumptions of Proposition 2.2.3. It follows that $C(Y / H)$, which is isometric to $C(Y) / H$, is $(\ln 3+\varepsilon)$-hyperbolic.

2.4. Contracting balls in a cone. In this section we assume that $Y$ is a proper metric space. The cone over $Y$ is contractible, nevertheless it is not necessarily locally contractible. To avoid this problem, we consider the following geometric assumption.

Condition H(l): For all $y \in Y$ and all $r \in \mathbb{R}_{+}$there exists a homotopy $h: \bar{B}(y, r) \times$ $[0,1] \rightarrow Y$ contracting the closed ball $\bar{B}(y, r)$ to $\{y\}$ with the following property: $\left|h\left(y^{\prime}, t\right)-y\right| \leqslant\left|y^{\prime}-y\right|+l$ for all $y^{\prime} \in \bar{B}(y, r)$ and all $t \in[0,1]$.

Proposition 2.4.1. Let $x$ be a point of the cone $C(Y)$ and $r_{1} \in \mathbb{R}^{+}$. If $Y$ satisfies the condition $\mathrm{H}(1)$, then the closed ball $\bar{B}\left(x, r_{1}\right)$ is contractible in $B\left(x, r_{1}+l\right)$.

Proof. We denote by $(y, r)$ the point $x$ and by $\bar{B}$ the closed ball $\bar{B}\left(x, r_{1}\right)$. We distinguish two cases.

Case 1 . If $r_{1} \geqslant r$, then the vertex of the cone $v$ belongs to $\bar{B}$. We consider the following homotopy.

$$
H: \bar{B} \times[0,1] \rightarrow C(Y), \quad\left(\left(y^{\prime}, r^{\prime}\right), t\right) \mapsto\left(y^{\prime},(1-t) r^{\prime}\right) .
$$

It contracts the ball $\bar{B}$ to the vertex $v$. Let $x^{\prime}=\left(y^{\prime}, r^{\prime}\right)$ be a point of $\bar{B}$. The metric in $\mathbb{H}_{2}$ is convex. Since the metric on the cone is modelled on the one on $\mathbb{H}_{2}$, we have for all $t \in[0,1]$,

$$
\left|H\left(x^{\prime}, t\right)-x\right|=\left|\left(y^{\prime},(1-t) r^{\prime}\right)-x\right| \leqslant \max \left\{\left|x^{\prime}-x\right|,|v-x|\right\} \leqslant r_{1} .
$$

Thus $H$ maps to $\bar{B}$.

Case 2. We assume now that $r_{1}<r$. It follows that $\left|y^{\prime}-y\right| \leqslant \pi \sinh r_{0}$ for all $\left(y^{\prime}, r^{\prime}\right) \in \bar{B}$. By assumption, there exists a homotopy map $h: \bar{B}\left(y, \pi \sinh r_{0}\right) \times$ 
$[0,1] \rightarrow Y$ contracting $\bar{B}\left(y, \pi \sinh r_{0}\right)$ to $\{y\}$ such that $\left|h\left(y^{\prime}, t\right)-y\right| \leqslant\left|y^{\prime}-y\right|+l$ for all $y^{\prime} \in \bar{B}\left(y, \pi \sinh r_{0}\right)$ and all $t \in[0,1]$. We consider the map

$$
H: \bar{B} \times[0,1] \rightarrow C(Y), \quad\left(\left(y^{\prime}, r^{\prime}\right), t\right) \mapsto\left(h\left(y^{\prime}, t\right), r^{\prime}\right) .
$$

It contracts $\bar{B}$ to $\{y\} \times\left[r-r_{1}, \min \left\{r_{0}, r+r_{1}\right\}\right]$. Let $x^{\prime}=\left(y^{\prime}, r^{\prime}\right)$ be a point of $\bar{B}$ and $t \in[0,1]$. By assumption, we have $\theta\left(y, h\left(y^{\prime}, t\right)\right) \leqslant \theta\left(y, y^{\prime}\right)+\alpha$, where $\alpha=\min \left\{\frac{l}{\sinh r_{0}}, \pi-\theta\left(y, y^{\prime}\right)\right\}$. The distances between $x, x^{\prime}$ and $H\left(x^{\prime}, t\right)$ may be viewed in $\mathbb{H}_{2}$. Due to the triangle inequality, we have

$$
\begin{aligned}
\left|H\left(x^{\prime}, t\right)-x\right|= & \operatorname{arccosh}\left(\cosh r \cosh r^{\prime}-\sinh r \sinh r^{\prime} \cos \theta\left(y, h\left(y^{\prime}, t\right)\right)\right) \\
\leq & \operatorname{arccosh}\left(\cosh r \cosh r^{\prime}-\sinh r \sinh r^{\prime} \cos \theta(y, y)\right) \\
& +\operatorname{arccosh}\left(\cosh ^{2} r^{\prime}-\sinh ^{2} r^{\prime} \cos \alpha\right) \\
\leq & \left|x^{\prime}-x\right|+\alpha \sinh r^{\prime} \leqslant r_{1}+l .
\end{aligned}
$$

Consequently, $H$ maps to $B\left(x, r_{1}+l\right)$. We conclude by noticing that $\{y\} \times\left[r-r_{1}\right.$, $\left.\min \left\{r_{0}, r+r_{1}\right\}\right]$ is contractible in $B\left(x, r_{1}+l\right)$.

The next lemma explains how to deformation retract a ball of the cone onto its base.

Proposition 2.4.2. Let $x=(y, r)$ be a point of the cone $C(Y)$ and let $\left.r_{1} \in\right] r_{0}-r, r[$. Assume that $Y$ satisfies the condition $\mathrm{H}(\mathrm{l})$. Then there exists a homotopy $H: \bar{B}\left(x, r_{1}\right) \times[0,1] \rightarrow \bar{B}\left(x, r_{1}+2 l\right)$ which contracts $\bar{B}\left(x, r_{1}\right)$ to a subset of $\iota(Y)$ and such that $H\left(x^{\prime}, t\right)=x^{\prime}$ for all $x^{\prime} \in \bar{B}\left(x, r_{1}\right) \cap \iota(Y)$ and all $t \in[0,1]$.

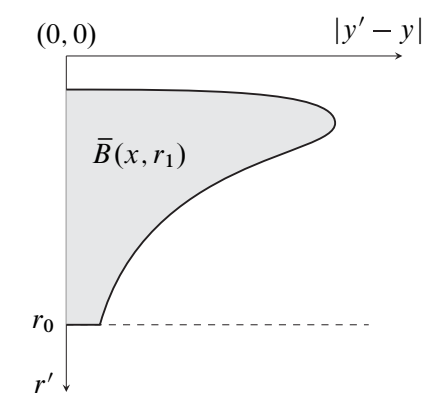

Figure 2. Shape of the ball $\bar{B}\left(x, r_{1}\right)$. The points $\left(y^{\prime}, r^{\prime}\right)$ that belong to $\bar{B}\left(x, r_{1}\right)$ lie in the gray part.

Remark. The first idea to prove this proposition is to project the cone onto its base, using the map $p$. Nevertheless, this homotopy does not stay in a neighbourhood of $\bar{B}\left(x, r_{1}\right)$. This problem can be observed on Figure 2 which represents the shape of 
$\bar{B}\left(x, r_{1}\right)$. In order to get around this difficulty, we have to proceed in two steps. At first, we contract the ball horizontally, using a homotopy of $Y$, then we project it onto the base.

Proof. Denote by $\bar{B}$ the closed ball $\bar{B}\left(x, r_{1}\right)$. Since $r_{1}<r$, we have $\left|y^{\prime}-y\right| \leqslant$ $\pi \sinh r_{0}$ for all points $x^{\prime}=\left(y^{\prime}, r^{\prime}\right)$ of $\bar{B}$. By assumption there exists a homotopy $h: \bar{B}\left(y, \pi \sinh r_{0}\right) \times[0,1] \rightarrow Y$ contracting $\bar{B}\left(y, \pi \sinh r_{0}\right)$ to $\{y\}$ such that $\left|h\left(y^{\prime}, t\right)-y\right| \leqslant\left|y^{\prime}-y\right|+l$ for all $y^{\prime} \in \bar{B}\left(y, \pi \sinh r_{0}\right)$ and all $t \in[0,1]$.

Let $x^{\prime}=\left(y^{\prime}, r^{\prime}\right)$ be a point of $C(Y)$. There exists a continuous function $L\left(r^{\prime}\right)=$ $\arccos \left(\frac{\cosh r^{\prime} \cosh r-\cosh r_{1}}{\sinh r^{\prime} \sinh r}\right)$ such that $x^{\prime}$ belongs to $\bar{B}$ if and only if $\left|y^{\prime}-y\right| \leqslant L\left(r^{\prime}\right)$. Since $r_{1}>r_{0}-r, L\left(r_{0}\right)$ is positive. By continuity, there exists $r_{2}<r_{0}$ such that $L\left(r^{\prime}\right) \leqslant L\left(r_{0}\right)+l$ for all $\left.r^{\prime} \in\right] r_{2}, r_{0}$ [. Since $h$ is continuous on a compact set, there exists $t_{0}$ such that $\left|h\left(y^{\prime}, t_{0}\right)-y\right| \leqslant L\left(r_{0}\right)$ for all $y^{\prime} \in \bar{B}\left(y, \pi \sinh r_{0}\right)$. We consider now the map

$$
H: \bar{B} \times[0,1] \rightarrow C(Y), \quad\left(\left(y^{\prime}, r^{\prime}\right), t\right) \rightarrow \begin{cases}\left(h\left(y^{\prime}, t t_{0}\right), r^{\prime}\right) & \text { if } r^{\prime} \leqslant r_{2}, \\ \left(h\left(y^{\prime}, \frac{r_{0}-r^{\prime}}{r_{0}-r_{2}} t t_{0}\right), r^{\prime}\right) & \text { if } r^{\prime}>r_{2} .\end{cases}
$$

The map $H$ is continuous. Furthermore, $H\left(x^{\prime}, t\right)=x^{\prime}$ for all $x^{\prime} \in \bar{B} \cap \iota(Y)$ and all $t \in[0,1]$. As in the previous proposition, we prove that $H$ maps to $B\left(x, r_{1}+l\right)$. We denote by $E$ the image of $\bar{B} \times\{1\}$ by $H$.

Lemma 2.4.3. The set $E$, the image by $H$ of $\bar{B} \times\{1\}$, has the following property. For all $x^{\prime}=\left(y^{\prime}, r^{\prime}\right) \in C(Y)$, if $x^{\prime}$ belongs to $E$, then $\left|y^{\prime}-y\right| \leqslant L\left(r_{0}\right)+2 l$.

Proof. Let $x^{\prime}=\left(y^{\prime}, r^{\prime}\right)$ be a point of $\bar{B}$. If $r^{\prime} \leqslant r_{2}$, then $H\left(x^{\prime}, 1\right)=\left(h\left(y^{\prime}, t_{0}\right), r^{\prime}\right)$. By construction of $t_{0}$, we have $\left|h\left(y^{\prime}, t_{0}\right)-y\right| \leqslant L\left(r_{0}\right)$. If $r^{\prime}>r_{2}$, then $H\left(x^{\prime}, 1\right)=$ $\left(h\left(y^{\prime}, \frac{r_{0}-r^{\prime}}{r_{0}-r_{2}} t_{0}\right), r^{\prime}\right)$. By definition of $r_{2}$ and $h$, we have

$$
\left|h\left(y^{\prime}, \frac{r_{0}-r^{\prime}}{r_{0}-r_{2}} t_{0}\right)-y\right| \leqslant\left|y^{\prime}-y\right|+l \leqslant L\left(r_{0}\right)+2 l .
$$

End of the proof of Proposition 2.4.2. We now consider a second homotopy

$$
H^{\prime}: E \times[0,1] \rightarrow C(Y), \quad\left(\left(y^{\prime}, r^{\prime}\right), t\right) \mapsto\left(y^{\prime},(1-t) r^{\prime}+t r_{0}\right) .
$$

The map $H^{\prime}$ contracts $E$ to a subset of $\iota(Y)$. Let $x^{\prime}=\left(y^{\prime}, r^{\prime}\right)$ be a point of $E \subset$ $B\left(x, r_{1}+l\right)$. By Lemma 2.4.3, $\left|y^{\prime}-y\right| \leqslant L\left(r_{0}\right)+2 l$. Thus $x^{\prime}$ and $\iota\left(y^{\prime}\right)$ are points of $B\left(x, r_{1}+2 l\right)$. The metric in $\mathbb{H}_{2}$ is convex. Since the metric on the cone is modelled on the one on $\mathbb{H}_{2}$, we have for all $t \in[0,1]$,

$\left|H^{\prime}\left(x^{\prime}, t\right)-x\right|=\left|\left(y^{\prime},(1-t) r^{\prime}+t r_{0}\right)-x\right| \leqslant \max \left\{\left|x^{\prime}-x\right|,\left|\iota\left(y^{\prime}\right)-x\right|\right\} \leqslant r_{1}+2 l$.

Thus $H^{\prime}$ maps to $B\left(x, r_{1}+2 l\right)$. Applying successively $H$ and $H^{\prime}$ provides the homotopy of the proposition. 


\section{Cone-off over a metric space}

The goal of this part is to study the large scale geometry of the cone-off. We give a detailed proof that under some small cancellation assumptions, the cone-off of a hyperbolic space is locally hyperbolic. We recall that $r_{0}$ is a fixed positive number whose value will be made precise in Section 4.

3.1. Definition. Let $X$ be a metric space and $Y=\left(Y_{i}\right)_{i \in I}$ a collection of subsets of $X$. For each $i \in I$, we consider the following objects: $C_{i}$ is the cone $C\left(Y_{i}\right)$ and $v_{i}$ its vertex; $\iota_{i}: Y_{i} \rightarrow C_{i}$ and $p_{i}: C_{i} \backslash\left\{v_{i}\right\} \rightarrow Y_{i}$ are the maps between the cone and its base defined in the previous part.

Definition 3.1.1 (Cone-off over a metric space). The cone-off over $X$ relatively to $Y$ is the space obtained by gluing each cone $C_{i}$ on $X$ along $Y_{i}$ according to $\iota_{i}$. We denote it by $\dot{X}(Y)$ (or simply $\dot{X}$ ).

Metric on the cone-off. In this paragraph we define a metric on the cone-off such that its restriction to a cone is the metric previously defined. To this end, one defines the metric as the lower bound of the length of chains joining two points. In this way, we obtain a pseudo-metric. The goal here is to prove that it is also positive.

We endow $X \sqcup\left(\bigsqcup_{i \in I} C_{i}\right)$ with the metric induced by $|\cdot|_{X}$ and $|\cdot|_{C_{i}}$. Let $x$ and $x^{\prime}$ be two points of $\dot{X}$. The quantity $\left\|x^{\prime}-x\right\|$ is the minimal distance between two points of $X \sqcup\left(\bigsqcup_{i \in I} C_{i}\right)$ whose image in $\dot{X}$ are respectively $x$ and $x^{\prime}$. The value of $\left\|x^{\prime}-x\right\|$ is different whether $x$ and $x^{\prime}$ both belong to a cone $C_{i}$ or not. The three possible cases are presented in the next lemma.

Recall that $\mu$ is the function defined in Proposition 2.1 .5 by

$$
\mu(t)=\operatorname{arccosh}\left(\cosh ^{2} r_{0}-\sinh ^{2} r_{0} \cos \left(\min \left\{\pi, \frac{t}{\sinh r_{0}}\right\}\right)\right) .
$$

It has the following interpretation. If $y$ and $y^{\prime}$ are two points of $Y_{i}$, then the distance between $\left(y, r_{0}\right)$ and $\left(y^{\prime}, r_{0}\right)$ in $C_{i}$ is $\mu\left(\left|y^{\prime}-y\right|_{X}\right)$.

Lemma 3.1.2. Let $x$ and $x^{\prime}$ be two points of $\dot{X}$.

(i) If there is $i \in I$ such that $x, x^{\prime} \in C_{i}$, then $\left\|x^{\prime}-x\right\|=\left|x^{\prime}-x\right|_{C_{i}}$. In particular, if $x, x^{\prime} \in Y_{i}$, then $\left\|x^{\prime}-x\right\|=\mu\left(\left|x^{\prime}-x\right|_{X}\right)$.

(ii) If $x, x^{\prime} \in X$, but there is no $i \in I$ such that $x, x^{\prime} \in Y_{i}$, then $\left\|x^{\prime}-x\right\|=$ $\left|x^{\prime}-x\right|_{X}$.

(iii) In all other cases, we have $\left\|x^{\prime}-x\right\|=+\infty$.

In particular, for all $x, x^{\prime} \in X,\left\|x^{\prime}-x\right\| \geqslant \mu\left(\left|x^{\prime}-x\right|_{X}\right)$.

The quantity $\left\|x^{\prime}-x\right\|$ does not define a metric. It does not indeed satisfy the triangle inequality. That is why we consider chains of points. 
Definition 3.1.3. Let $x$ and $x^{\prime}$ be two points of $\dot{X}$.

A chain between $x$ and $x^{\prime}$ is a finite sequence $C=\left(z_{1}, \ldots, z_{n}\right)$ of points of $\dot{X}$, such that $z_{1}=x$ and $z_{m}=x^{\prime}$. Its length is $l(C)=\sum_{j=1}^{m-1}\left\|z_{j+1}-z_{j}\right\|$.

Furthermore we define

$$
\left|x^{\prime}-x\right|_{\dot{X}}=\inf \left\{l(C) \mid C \text { a chain between } x \text { and } x^{\prime}\right\} .
$$

Obviously, $|\cdot|_{\dot{X}}$ is a pseudo-metric.

Lemma 3.1.4. Let $x$ and $x^{\prime}$ be two points of $\dot{X}$. For all $\varepsilon>0$ there is a chain $C=\left(z_{1}, \ldots, z_{m}\right)$ between $x$ and $x^{\prime}$ satisfying the following.

(i) $l(C) \leqslant\left|x^{\prime}-x\right|_{\dot{X}}+\varepsilon$.

(ii) $z_{j} \in X$ for all $j \in\{2, \ldots, m-1\}$.

Proof. Let $\varepsilon>0$. By definition there exists $C=\left(z_{1}, \ldots z_{m}\right)$ a chain between $x$ and $x^{\prime}$ such that $l(C) \leqslant\left|x^{\prime}-x\right|_{X}+\varepsilon$. Assume that there is $j \in\{2, \ldots, m-1\}$ such that $z_{j}$ does not belong to $X$. It follows that $z_{j}$ is strictly contained in a cone, that is there exists $i \in I$ such that $z_{j} \in C_{i} \backslash \iota_{i}\left(Y_{i}\right)$. In particular there is only one point of $\left(\bigsqcup_{i \in I} C_{i}\right) \sqcup X$ whose image in $\dot{X}$ is $z_{j}$. Using the triangle inequality in $C_{i}$, we have $\left\|z_{j+1}-z_{j-1}\right\| \leqslant\left\|z_{j+1}-z_{j}\right\|+\left\|z_{j}-z_{j-1}\right\|$. Thus the sequence $C^{\prime}$ obtained by removing the point $z_{j}$ from $C$ is a chain between $x$ and $x^{\prime}$ shorter than $C$. Hence after removing all points of $C$ which are not in $X$, we obtain a chain satisfying the properties of the lemma.

Lemma 3.1.5. For all $i \in I,|\cdot|_{C_{i}}$ and $|\cdot|_{\dot{X}}$ locally coincide: Let $x=(y, r)$ be a point of $C_{i} \backslash \iota_{i}\left(Y_{i}\right)$. If $x^{\prime}$ is a point of $\dot{X}$ such that $\left|x^{\prime}-x\right|_{\dot{X}} \leqslant \frac{1}{4}\left|r_{0}-r\right|$, then $x^{\prime} \in C_{i}$ and $\left|x^{\prime}-x\right|_{\dot{X}}=\left|x^{\prime}-x\right|_{C_{i}}$.

Proof. Let $i \in I$ and $x=(y, r)$ be a point of $C_{i} \backslash \iota_{i}\left(Y_{i}\right)$. Since $x \notin \iota_{i}\left(Y_{i}\right), r_{0}-r>0$. Let $x^{\prime}$ be a point of $\dot{X}$ such that $\left|x^{\prime}-x\right|_{\dot{X}} \leqslant \frac{1}{4}\left(r_{0}-r\right)$. Let $\left.\eta \in\right] 0, \frac{1}{4}\left(r_{0}-r\right)[$. Using the previous lemma, there is a chain $C=\left(z_{1}, \ldots, z_{m}\right)$ between $x$ and $x^{\prime}$ such that $l(C) \leqslant\left|x^{\prime}-x\right|_{\dot{X}}+\eta$ and for all $j \in\{2, \ldots, m-1\}, z_{j} \in X$. Assume that $m \geqslant 3$. Since $x \in C_{i} \backslash \iota_{i}\left(Y_{i}\right)$, we have

$$
r_{0}-r \leqslant\left\|z_{2}-z_{1}\right\| \leqslant l(C) \leqslant\left|x^{\prime}-x\right|_{\dot{X}}+\eta \leqslant \frac{1}{2}\left(r_{0}-r\right) .
$$

Contradiction. Thus $m=2$ and $\left\|x^{\prime}-x\right\|=l(C) \leqslant \frac{1}{2}\left(r_{0}-r\right)$. Consequently $x^{\prime}$ belongs to $C_{i}$ and $\left\|x^{\prime}-x\right\|=\left|x^{\prime}-x\right|_{C_{i}}$. Hence for all $\left.\eta \in\right] 0, \frac{1}{4}\left(r_{0}-r\right)[$, we have

$$
\left|x^{\prime}-x\right|_{\dot{X}} \leqslant\left|x^{\prime}-x\right|_{C_{i}} \leqslant\left|x^{\prime}-x\right|_{\dot{X}}+\eta \text {. }
$$

It follows that $\left|x^{\prime}-x\right|_{\dot{X}}=\left|x^{\prime}-x\right|_{C_{i}}$.

Lemma 3.1.6. For all $x, x^{\prime} \in X$, we have $\left|x^{\prime}-x\right|_{\dot{X}} \geqslant \mu\left(\left|x^{\prime}-x\right|_{X}\right)$. 
Proof. Let $\varepsilon>0$. Using Lemma 3.1.4, there exists a chain $C=\left(z_{1}, \ldots, z_{m}\right)$ between $x$ and $x^{\prime}$ such that $l(C) \leqslant\left|x^{\prime}-x\right|_{\dot{X}}+\varepsilon$ and for all $j \in\{1, \ldots, m\}, z_{j} \in X$. The subadditivity of $\mu$ gives

$$
\mu\left(\left|x^{\prime}-x\right|_{X}\right) \leqslant \sum_{j=1}^{m-1} \mu\left(\left|z_{j+1}-z_{j}\right|\right) \leqslant \sum_{j=1}^{m-1}\left\|z_{j+1}-z_{j}\right\|=l(C) .
$$

Thus for all $\varepsilon>0$, we have $\mu\left(\left|x^{\prime}-x\right|_{X}\right) \leqslant\left|x^{\prime}-x\right|_{\dot{X}}+\varepsilon$. It follows that $\left|x^{\prime}-x\right|_{\dot{X}} \geqslant$ $\mu\left(\left|x^{\prime}-x\right|_{X}\right)$.

Proposition 3.1.7. $|\cdot|_{\dot{X}}$ defines a metric on $\dot{X}$.

Proof. The only point to prove is the positivity of $|\cdot|_{\dot{X}}$. Consider two points $x$ and $x^{\prime}$ of $\dot{X}$ such that $\left|x^{\prime}-x\right|_{\dot{X}}=0$. There are two cases.

(i) If there is $i \in I$ such that $x \in C_{i} \backslash \iota_{i}\left(Y_{i}\right)$ or $x^{\prime} \in C_{i} \backslash \iota_{i}\left(Y_{i}\right)$, then, using Lemma 3.1.5, $x$ and $x^{\prime}$ both belong to $C_{i}$. Moreover $\left|x^{\prime}-x\right|_{C_{i}}=\left|x^{\prime}-x\right|_{\dot{X}}=0$. Thus $x=x^{\prime}$.

(ii) If $x$ and $x^{\prime}$ are both elements of $X$, then, due to Lemma 3.1.6, we have $\mu\left(\left|x^{\prime}-x\right|_{X}\right) \leqslant\left|x^{\prime}-x\right|_{\dot{X}}=0$. Hence $\left|x^{\prime}-x\right|_{X}=0$. It follows that $x=x^{\prime}$.

Projection of the cone-off on its base. We consider a map $p$ from $\dot{X} \backslash\left\{v_{i}, i \in I\right\}$ onto $X$ whose restriction to a cone $C_{i} \backslash\left\{v_{i}\right\}$ is $p_{i}$ and whose restriction to $X$ is the identity.

Proposition 3.1.8. Consider a point $x$ of $\dot{X}$ such that the distance between $x$ and any vertex of $\dot{X}$ is at least $\frac{r_{0}}{2}$. For all $x^{\prime} \in B\left(x, \frac{r_{0}}{3}\right)$, we have $\left|p\left(x^{\prime}\right)-p(x)\right|_{X} \leqslant$ $\frac{3 \pi \sinh r_{0}}{r_{0}}\left|x^{\prime}-x\right|_{\dot{X}}$.

Proof. Let $\varepsilon \in] 0, \frac{r_{0}}{2}\left[\right.$. Consider a point $x^{\prime}$ of $B\left(x, \frac{r_{0}}{3}\right)$. Using Lemma 3.1.4, there exists a chain $C=\left(z_{1}, \ldots, z_{m}\right)$ such that for all $j$ belonging to $\{2, \ldots, m-1\}, z_{j} \in X$ and $l(C) \leqslant\left|x^{\prime}-x\right|_{\dot{X}}+\varepsilon \leqslant r_{0}$. We choose $j \in\{2, \ldots, m-1\}$. Lemma 2.1.5 gives

$$
\begin{aligned}
r_{0} \geqslant l(C) & \geqslant\left\|z_{j+1}-z_{j}\right\| \geqslant \mu\left(\left|z_{j+1}-z_{j}\right|_{X}\right) \\
& \geqslant \frac{2 r_{0}}{\pi \sinh r_{0}} \min \left\{\pi \sinh r_{0},\left|z_{j+1}-z_{j}\right|_{X}\right\} .
\end{aligned}
$$

Thus $\left|p\left(z_{j+1}\right)-p\left(z_{j}\right)\right|_{X}=\left|z_{j+1}-z_{j}\right|_{X} \leqslant \frac{3 \pi \sinh r_{0}}{r_{0}}\left\|z_{j+1}-z_{j}\right\|$.

If $x=z_{1}$ is a point of $X$, by same proof we obtain $\left|p\left(z_{2}\right)-p\left(z_{1}\right)\right|_{X} \leqslant$ $\frac{3 \pi \sinh r_{0}}{r_{0}}\left\|z_{2}-z_{1}\right\|$. On the other hand, if $x$ belongs to a cone $C_{i}$, then Lemma 2.1.4 gives the same inequality. In the same way, we obtain

$$
\left|p\left(z_{m}\right)-p\left(z_{m-1}\right)\right|_{X} \leqslant \frac{3 \pi \sinh r_{0}}{r_{0}}\left\|z_{m}-z_{m-1}\right\| .
$$


Consequently, we have

$$
\begin{aligned}
\left|p^{\prime}(x)-p(x)\right|_{X} \leqslant \sum_{j=1}^{m-1}\left|p\left(z_{j+1}\right)-p\left(z_{j}\right)\right|_{X} & \leqslant \frac{3 \pi \sinh r_{0}}{r_{0}} \sum_{j=1}^{m-1}\left\|z_{j+1}-z_{j}\right\| \\
& =\frac{3 \pi \sinh r_{0}}{r_{0}} l(C) \\
& \leqslant \frac{3 \pi \sinh r_{0}}{r_{0}}\left(\left|x^{\prime}-x\right|_{\dot{X}}+\varepsilon\right) .
\end{aligned}
$$

Hence $\left|p^{\prime}(x)-p(x)\right|_{X} \leqslant \frac{3 \pi \sinh r_{0}}{r_{0}}\left|x^{\prime}-x\right|_{\dot{X}}$.

3.2. Uniform approximation of the distance on the cone-off. In order to study the ultra limit of a sequence of cone-off spaces, we need to approximate the distance between two points of $\dot{X}$ by a chain such that the number of points involved in the chain only depends on the error and not on the base space $X$. This point was already noted by M. Gromov in [14]. More precisely, in this section we prove the following result:

Proposition 3.2.1. Let $A \geqslant 1$. There exists a constant $M$, depending only on $A$ and not on $r_{0}$, with the following property. Let $\left.\varepsilon \in\right] 0,1[, X$ be a metric space and $Y=\left(Y_{i}\right)_{i \in I}$ a collection of subsets of $X$. Let $x$ and $x^{\prime}$ be two points of the cone-off $\dot{X}(Y)$ such that $\left|x^{\prime}-x\right|_{\dot{X}} \leqslant A$. There exists a chain $C$ between $x$ and $x^{\prime}$ with less than $\frac{M}{\sqrt{\varepsilon}}$ points and such that $l(C) \leqslant\left|x^{\prime}-x\right|_{\dot{X}}+\varepsilon$.

Proof. Let $\varepsilon \in] 0,1\left[\right.$. Let $x$ and $x^{\prime}$ be two points of $\dot{X}$ such that $\left|x^{\prime}-x\right|_{\dot{X}} \leqslant A$. Using Lemma 3.1.4, there is a chain $C=\left(z_{1}, \ldots, z_{n}\right)$ between $x$ and $x^{\prime}$ such that $l(C) \leqslant\left|x^{\prime}-x\right|_{\dot{X}}+\frac{1}{2} \varepsilon$ and for all $j \in\{2, \ldots, n-1\}, z_{j} \in X$. We fix $\left.\eta \in\right] 0,1[$, and construct a subchain of $C$ between $x$ and $x^{\prime}$, denoted by $C_{\eta}=\left(z_{j_{1}}, \ldots, z_{j_{m}}\right)$, as follows.

(i) Put $j_{1}=1$ and $j_{2}=2$.

(ii) Let $k \geqslant 2$. We construct $j_{k+1}$ from $j_{k}$.

If $j_{k}<n-1$ and $\left|z_{j_{k}+1}-z_{j_{k}}\right|_{X}>\eta$, then $j_{k+1}=j_{k}+1$.

If $j_{k}<n-1$ and $\left|z_{j_{k}+1}-z_{j_{k}}\right|_{X} \leqslant \eta$, then $j_{k+1}$ is the largest $j \in\left\{j_{k}+1, \ldots, n-\right.$ $1\}$ such that $\left|z_{j}-z_{j_{k}}\right|_{X} \leqslant \eta$.

If $j_{k}=n-1$, then $j_{k+1}=n$ and the process stops.

This construction removes from $C$ the small parts of the chain which may be contained in a cone. We prove now that it hardly changes the length of the chain.

Lemma 3.2.2 (Comparison between the two chains). The chains $C_{\eta}$ and $C$ satisfy the inequality $l\left(C_{\eta}\right) \leqslant l(C)+m \eta^{3}$, where $m$ is the number of points in $C_{\eta}$.

Proof. We consider an integer $k \in\{1, \ldots, m-2\}$. There are two cases. 
First case: Assume that $\left|z_{j_{k+1}}-z_{j_{k}}\right| x \leqslant \eta$. The function $\mu$ given by Proposition 2.1.5 has the following property: $\mu(t) \geqslant t-t^{3}$ for all $t \in\left[0, \pi \sinh r_{0}\right]$. Thus using the subadditivity of $\mu$ we obtain

$$
\begin{aligned}
\sum_{j=j_{k}}^{j_{k+1}-1}\left\|z_{j+1}-z_{j}\right\| & \geqslant \sum_{j=j_{k}}^{j_{k+1}-1} \mu\left(\left|z_{j+1}-z_{j}\right|_{X}\right) \\
& \geqslant \mu\left(\left|z_{j_{k+1}}-z_{j_{k}}\right| X\right) \geqslant\left|z_{j_{k+1}}-z_{j_{k}}\right|_{X}-\left|z_{j_{k+1}}-z_{j_{k}}\right|_{X}^{3} .
\end{aligned}
$$

Thus we have $\sum_{j=j_{k}}^{j_{k+1}-1}\left\|z_{j+1}-z_{j}\right\| \geqslant\left\|z_{j_{k+1}}-z_{j_{k}}\right\|-\eta^{3}$.

Second case: Assume that $\left|z_{j_{k+1}}-z_{j_{k}}\right|_{X}>\eta$. By construction, we have $j_{k+1}=$ $j_{k}+1$. Hence the last inequality remains true.

After summing over $k$ these inequalities, we finally obtain $l(C) \geqslant l\left(C_{\eta}\right)-m \eta^{3}$.

Lemma 3.2.3 (Estimation of $m$ ). If $\eta \leqslant \frac{1}{4}$, then $m$, the number of points in the chain $C_{\eta}$, is less than $100 \frac{A}{\eta}$.

Proof. Let $k \in\{2, \ldots, m-3\}$. The two inequalities $\left|z_{j_{k+1}}-z_{j_{k}}\right|_{X} \leqslant \frac{1}{2} \eta$ and $\left|z_{j_{k+2}}-z_{j_{k+1}}\right| x \leqslant \frac{1}{2} \eta$ cannot be both true. Indeed, if it was the case, $j_{k+1}$ will not be the largest $j \in\left\{j_{k}+1, \ldots, n-1\right\}$ such that $\left|z_{j}-z_{j_{k}}\right|_{X} \leqslant \eta$. Assume that $\left|z_{j_{k+1}}-z_{j_{k}}\right| X>\frac{1}{2} \eta$ (the other case is symmetric). Using the same estimation of $\mu$ as the one in the previous lemma, we obtain

$$
\left\|z_{j_{k+1}}-z_{j_{k}}\right\| \geqslant \mu\left(\left|z_{j_{k+1}}-z_{j_{k}}\right| X\right) \geqslant \mu\left(\frac{1}{2} \eta\right) \geqslant \frac{1}{2} \eta-\frac{1}{8} \eta^{3} .
$$

Thus $\left\|z_{j_{k+1}}-z_{j_{k}}\right\|+\left\|z_{j_{k+2}}-z_{j_{k+1}}\right\| \geqslant \frac{1}{2} \eta-\frac{1}{8} \eta^{3}$. After summing over $k$, the previous lemma gives

$$
\left\lfloor\frac{m-4}{2}\right\rfloor\left(\frac{1}{2} \eta-\frac{1}{8} \eta^{3}\right) \leqslant l\left(C_{\eta}\right) \leqslant l(C)+m \eta^{3} \leqslant\left|x^{\prime}-x\right|_{\dot{X}}+\frac{1}{2} \varepsilon+m \eta^{3} .
$$

We have the inequality

$$
m\left(4-17 \eta^{2}\right) \leqslant 50 \frac{A}{\eta} .
$$

Hence if $\eta \leqslant \frac{1}{4}$, then $4-17 \eta^{2} \geqslant \frac{1}{2}$. It follows that $m$ must be bounded by $100 \frac{A}{\eta}$.

End of the proof of Proposition 3.2.1. Combining the two previous lemmas, we obtain

$$
l\left(C_{\eta}\right) \leqslant l(C)+m \eta^{3} \leqslant\left|x^{\prime}-x\right|_{\dot{X}}+\frac{1}{2} \varepsilon+100 A \eta^{2} .
$$

If we choose $\eta=\frac{1}{10} \sqrt{\frac{\varepsilon}{2 A}}$, then we have $l\left(C_{\eta}\right) \leqslant\left|x^{\prime}-x\right|_{\dot{X}}+\varepsilon$. Moreover the number $m$ of points of $C_{\eta}$ is less than $1000 A \sqrt{\frac{2 A}{\varepsilon}}$. 
3.3. Contracting balls of the cone-off. In this section $X$ is a proper, geodesic, $\delta$-hyperbolic space. We consider a collection $Y=\left(Y_{i}\right)_{i \in I}$ of closed strongly quasiconvex subsets of $X$. We assume that $X$ satisfies the condition $\mathrm{H}(\mathrm{l})$, i.e.,

Condition H(l): For all $x \in X$ and $r \in \mathbb{R}^{+}$, there exists a homotopy $h: \bar{B}(x, r) \times$ $[0,1] \rightarrow X$ which contracts the closed ball $\bar{B}(x, r)$ to $\{x\}$ such that $\left|h\left(x^{\prime}, t\right)-x\right| \leqslant$ $\left|x^{\prime}-x\right|+l$ for all $x^{\prime} \in \bar{B}(x, r)$ and all $t \in[0,1]$.

We also assume that the $Y_{i}$ 's satisfy the same condition.

Proposition 3.3.1. Let $x$ be a point of $\dot{X}$ and $r \in \mathbb{R}^{+}$. We assume that $\left|v_{i}-x\right|>r$ for all $i \in I$. Then the closed ball $\bar{B}(x, r)$ is contractible in $B(x, r+3 l)$.

Proof. If there exists $i \in I$ such that $\bar{B}(x, r)$ is contained in a cone $C\left(Y_{i}\right)$, then we apply Proposition 2.4.1. Otherwise we proceed as follows. Let $i \in I$. Assume that $\bar{B}(x, r) \cap C\left(Y_{i}\right) \neq \emptyset$. By Proposition 2.4.2, there exists a homotopy $H_{i}: \bar{B}(x, r) \cap$ $C\left(Y_{i}\right) \times[0,1] \rightarrow B(x, r+2 l)$ satisfying the following properties.

(i) $H_{i}$ contracts $\bar{B}(x, r) \cap C\left(Y_{i}\right)$ to a subset of $Y_{i}$.

(ii) $H_{i}\left(x^{\prime}, t\right)=x^{\prime}$ for all $x^{\prime} \in \bar{B}(x, r) \cap Y_{i}$ and all $t \in[0,1]$.

Thus we may define a map $H: \bar{B}(x, r) \times[0,1] \rightarrow B(x, r+2 l)$ such that its restriction to $\bar{B}(x, r) \cap C\left(Y_{i}\right) \times[0,1]$ is $H_{i}$, and $H\left(x^{\prime}, t\right)=x^{\prime}$ for all $x^{\prime} \in \bar{B}(x, r) \cap X$ and all $t \in[0,1]$. It follows that $H$ contracts $\bar{B}(x, r)$ to a subset of $B(x, r+2 l) \cap X$. By condition $\mathrm{H}(1)$, this set is contractible in $B(x, r+3 l)$.

\subsection{Hyperbolicity of the cone-off over an $\mathbb{R}$-tree}

Proposition 3.4.1. Let $X$ be an $\mathbb{R}$-tree and $Y=\left(Y_{i}\right)_{i \in I}$ a collection of subtrees of $X$ such that two distinct elements of $Y$ share no more than one point. The cone-off $\dot{X}(Y)$ is $\ln$ 3-hyperbolic.

Remark. In fact $\dot{X}(Y)$ is a CAT(-1) space, but we shall not use this point.

This result is a consequence of the more particular case where $X$ is a finite simplicial tree.

Lemma 3.4.2. Consider afinite simplicial tree $X$ and a finite collection $Y=\left(Y_{i}\right)_{i \in I}$ of subtrees of $X$ such that two distinct elements of $Y$ share no more than one point.

The cone-off $\dot{X}(Y)$ is CAT(-1). In particular it is $\ln 3$-hyperbolic.

Proof. Each $Y_{i}$ is a tree, thus all the cones $C\left(Y_{i}\right)$ are CAT(-1) (cf. Lemma 2.2.2). Consequently the cone-off $\dot{X}$ is obtained by gluing a finite number of CAT $(-1)$-spaces that share no more than one point. These spaces are the cones and the remaining parts of $X$ on which no cone is glued. It follows that $\dot{X}$ is CAT(-1) (cf [5], Chap. II.11, Th. 11.1). 
Proof of Proposition 3.4.1. Let $x, y, z$ and $t$ be four points of $\dot{X}$. For all $n \in \mathbb{N}$, we can find a finite simplicial subtree $X_{n}$ of $X$ and a finite collection $Y^{n}$ of subtrees of $X_{n}$ such that

- two distinct elements of $Y^{n}$ share no more than one point,

- $x, y, z, t$ belong to $\dot{X}_{n}\left(Y^{n}\right)$,

- for all $u, v \in\{x, y, z, t\}$, we have $\lim _{n \rightarrow+\infty}|v-u|_{\dot{X}_{n}\left(Y_{n}\right)}=|v-u|_{\dot{X}(Y)}$.

This can be done in the following way. Let $n \in \mathbb{N}$. For all pair of points in $\{x, y, z, t\}$ we consider a chain which approximates the distance between them, with an error smaller than $\frac{1}{n}$. These chains contain a finite number of points. Thus we choose $X_{n}$ and $Y^{n}$ such that the chains have the same length in $\dot{X}(Y)$ and $\dot{X}_{n}\left(Y^{n}\right)$. Since $\dot{X}_{n}$ is $\ln$ 3-hyperbolic (see Lemma 3.4.2), $x, y, z, t$ satisfy in these spaces the hyperbolicity condition. After taking the limit, we obtain in $\dot{X}$ the inequality $\langle x, z\rangle_{t} \geqslant \min \left\{\langle x, y\rangle_{t},\langle y, z\rangle_{t}\right\}-\ln 3$.

3.5. Hyperbolicity of the cone-off over a hyperbolic space. In this section, we generalize the previous proposition when the base $X$ is a hyperbolic space. Let $\delta$ be a positive number. We consider a geodesic, $\delta$-hyperbolic space $X$ and a collection $Y=\left(Y_{i}\right)_{i \in I}$ of closed $10 \delta$-quasi-convex subsets of $X$. In order to estimate the hyperbolicity of $\dot{X}(Y)$, we define a constant which controls how much two elements of $Y$ overlap.

Definition 3.5.1. The largest piece of $Y$, denoted by $\Delta(Y)$, is the quantity

$$
\Delta(Y)=\sup _{i \neq j} \operatorname{diam}\left(Y_{i}^{+20 \delta} \cap Y_{j}^{+20 \delta}\right) .
$$

Assume that $X$ is an $\mathbb{R}$-tree, so that $\delta=0$. Then $\Delta(Y)$ is zero if and only if two distinct elements of $Y$ share no more than one point.

Theorem 3.5.2 (Second hyperbolicity theorem). Let $\varepsilon>0$. There exist $\delta, \Delta>0$ satisfying the following properties. Consider a geodesic, $\delta$-hyperbolic space $X$ and a collection $Y=\left(Y_{i}\right)_{i \in I}$ of closed, 108-quasi-convex subsets of $X$ such that $\Delta(Y) \leqslant$ $\Delta$. If $x_{0}$ is a point of the cone-off $\dot{X}(Y)$ whose distance to any vertex is greater than $\frac{r_{0}}{2}$, then the ball $B\left(x_{0}, \frac{r_{0}}{9}\right)$ is $(\ln 3+\varepsilon)$-hyperbolic.

Remark. This theorem is an extension of Proposition 3.4.1 for spaces that may be viewed as $\delta$-perturbed $\mathbb{R}$-trees. Thus it is possible to prove that $\dot{X}(Y)$ satisfies the CAT(-1)-condition with a small error depending only on $\delta$ and $\Delta$. M. Gromov introduced in [14] the notion of CAT $(-1, \varepsilon)$-spaces which formalizes this idea. It was also developed in [8]. Since we are only interested in the hyperbolicity of $\dot{X}(Y)$, we will not use it.

In [14], M. Gromov gave a quantitative statement (Hyperbolic Coning Lemma) of this result. We propose here a detailed proof which provides a qualitative version 
of the theorem. The strategy is as follows. Assuming that this theorem is false gives a family $X_{n}$ of $\delta_{n}$-hyperbolic counter-examples with $\delta_{n}$ tending to zero. Taking the limit gives the cone off over an $\mathbb{R}$-tree which we already know to be ln 3-hyperbolic. This is a contradiction according to Corollary 1.1.4. The point is to construct a local isometry between the cone-off over the ultra-limit of $\left(X_{n}\right)$ and the ultra-limit of the cones-off over $X_{n}$.

Proof. Assume that the theorem is false. Then for all $n \in \mathbb{N}$, we can find

(i) a geodesic, $\delta_{n}$-hyperbolic space $X_{n}$, with $\delta_{n}=o(1)$,

(ii) a collection $Y_{n}=\left(Y_{n, i_{n}}\right)_{i_{n} \in I_{n}}$ of closed, $10 \delta_{n}$-quasi-convex subsets of $X_{n}$, with $\Delta\left(Y_{n}\right)=o(1)$

(iii) a point $x_{n}^{0} \in \dot{X}_{n}\left(Y_{n}\right)$ whose distance to any vertex is greater than $\frac{r_{0}}{2}$ and such that the ball $B\left(x_{n}^{0}, \frac{r_{0}}{9}\right)$ is not $(\ln 3+\varepsilon)$-hyperbolic.

We fix a non-principal ultra-filter $\omega$ in order to study the $\operatorname{limit}_{\text {space }} \lim _{\omega}\left(\dot{X}_{n}, x_{n}^{0}\right)$. First we consider several objects.

- $x^{0}=\lim _{\omega} x_{n}^{0}$

- $X=\lim _{\omega}\left(X_{n}, p_{n}\left(x_{n}^{0}\right)\right)$ (recall that $p_{n}$ is the projection from $\dot{X}_{n}$ onto $\left.X_{n}\right)$;

- $I=\prod_{n \in \mathbb{N}} I_{n} / \sim$ where $\sim$ is the equivalence relation defined by $i \sim j$ if $i_{n}=j_{n} \omega$-as;

- if $i$ is a sequence of $\prod_{n \in \mathbb{N}} I_{n}$, we define $Y_{i}=\lim _{\omega} Y_{n, i_{n}}$.

Lemma 3.5.3. Let $i=\left(i_{n}\right)$ and $j=\left(j_{n}\right)$ be two sequences of $\prod_{n \in \mathbb{N}} I_{n}$. If $i_{n}=j_{n}$ $\omega$-as, then $Y_{i}=Y_{j}$; otherwise, $\operatorname{diam}\left(Y_{i} \cap Y_{j}\right)=0$.

Proof. If $i_{n}=j_{n} \omega$-as, the equality $Y_{i}=Y_{j}$ follows from the definition of the $\omega$-limit of a sequence of subsets. In the other case, we have $i_{n} \neq j_{n} \omega$-as. Hence $\operatorname{diam}\left(Y_{n, i_{n}}^{+20 \delta_{n}} \cap Y_{n, j_{n}}^{+20 \delta_{n}}\right) \leqslant \Delta_{n} \omega$-as. Thus Corollary 1.2.5 gives $\operatorname{diam}\left(Y_{i} \cap Y_{j}\right) \leqslant$ $\lim _{\omega} \operatorname{diam}\left(Y_{n, i_{n}}^{+20 \delta_{n}} \cap Y_{n, j_{n}}^{+20 \delta_{n}}\right)=0$.

Due to the previous lemma, we may consider the collection $Y=\left(Y_{i}\right)_{i \in I}$.

Lemma 3.5.4. The cone-off $\dot{X}(Y)$ is $\ln$ 3-hyperbolic.

Proof. Since for all $n \in \mathbb{N}, X_{n}$ is geodesic and $\delta_{n}$-hyperbolic with $\delta_{n}=o(1), X$ is an $\mathbb{R}$-tree. Furthermore, any $Y_{n, i_{n}}$ is a $10 \delta_{n}$-quasi-convex subset of $X_{n}$. By the previous lemma, $Y$ is a collection of subtrees such that two distinct elements of $Y$ share no more than one point. Applying Proposition 3.4.1, $\dot{X}(Y)$ is $\ln$ 3-hyperbolic.

The next step is to produce a local isometry between $\dot{X}(Y)$, the cone-off over $\lim _{\omega} X_{n}$, and $\lim _{\omega}\left(\dot{X}_{n}, x_{n}^{0}\right)$. For this purpose we consider the maps

$$
\begin{aligned}
\psi: X \rightarrow \lim _{\omega} \dot{X}_{n}, \quad \lim _{\omega} x_{n} \mapsto \lim _{\omega} x_{n}, \\
\psi_{i}: C\left(Y_{i}\right) \rightarrow \lim _{\omega} \dot{X}_{n}, \quad\left(\lim _{\omega} y_{n}, r\right) \mapsto \lim _{\omega}\left(y_{n}, r\right) .
\end{aligned}
$$


These maps induce a function $\dot{\psi}$ from $\dot{X}$ to $\lim _{\omega} \dot{X}_{n}$ such that its restriction to $X$ (resp. $C\left(Y_{i}\right)$ ) is $\psi$ (resp. $\psi_{i}$ ). At first we prove that this map is 1-Lipschitz, then we show that it induces a local isometry.

Lemma 3.5.5. Let $x$ and $x^{\prime}$ be two points of $\dot{X}$. We have $\left\|x^{\prime}-x\right\| \geqslant\left|\dot{\psi}\left(x^{\prime}\right)-\dot{\psi}(x)\right|$.

Proof. We distinguish three cases.

(i) Assume that there is $i \in I$ such that $x, x^{\prime} \in C\left(Y_{i}\right)$. Then we can write $x=(y, r)$ and $x^{\prime}=\left(y^{\prime}, r^{\prime}\right)$, where $y=\lim _{\omega} y_{n}$ and $y^{\prime}=\lim _{\omega} y_{n}^{\prime}$ are two points of $Y_{i}$. In this situation we have

$$
\left\|x^{\prime}-x\right\|=\left|x^{\prime}-x\right|_{C\left(Y_{i}\right)}=\operatorname{arccosh}\left(\cosh r \cosh r^{\prime}-\sinh r \sinh r^{\prime} \cos \theta\left(y, y^{\prime}\right)\right) .
$$

By continuity, This gives

$$
\begin{aligned}
\left\|x^{\prime}-x\right\| & =\lim _{\omega} \operatorname{arccosh}\left(\cosh r \cosh r^{\prime}-\sinh r \sinh r^{\prime} \cos \theta\left(y_{n}, y_{n}^{\prime}\right)\right) \\
& =\lim _{\omega}\left|\left(y_{n}^{\prime}, r^{\prime}\right)-\left(y_{n}, r\right)\right|_{C\left(Y_{n, i_{n}}\right)} \\
& \geqslant \lim _{\omega}\left|\left(y_{n}^{\prime}, r^{\prime}\right)-\left(y_{n}, r\right)\right|_{\dot{X}_{n}} \\
& =\left|\dot{\psi}\left(x^{\prime}\right)-\dot{\psi}(x)\right| .
\end{aligned}
$$

(ii) Assume that $x=\lim _{\omega} x_{n}$ and $x^{\prime}=\lim _{\omega} x_{n}^{\prime}$ belong to $X$, but there is no $i \in I$ such that $x, x^{\prime} \in Y_{i}$. In this case $\left\|x^{\prime}-x\right\|=\left|x^{\prime}-x\right|_{X}=\lim _{\omega}\left|x_{n}^{\prime}-x_{n}\right|_{X_{n}}$. However, for all $n \in \mathbb{N}$, we have $\left|x_{n}^{\prime}-x_{n}\right|_{X_{n}} \geqslant\left|x_{n}^{\prime}-x_{n}\right|_{\dot{X}_{n}}$. Thus $\left\|x^{\prime}-x\right\| \geqslant$ $\lim _{\omega}\left|x_{n}^{\prime}-x_{n}\right|_{\dot{X}_{n}}=\left|\dot{\psi}\left(x^{\prime}\right)-\dot{\psi}(x)\right|$.

(iii) In all other cases, $\left\|x^{\prime}-x\right\|=+\infty$. There is nothing to prove.

Corollary 3.5.6. The map $\dot{\psi}: \dot{X} \rightarrow \lim _{\omega} \dot{X}_{n}$ is 1-Lipschitz, where $\dot{X}$ is the cone-off over $\lim _{\omega} X_{n}$.

Proof. Let $x$ and $x^{\prime}$ be two points of $\dot{X}$. Consider a chain $C=\left(z_{1}, \ldots z_{m}\right)$ of points of $\dot{X}$ between $x$ and $x^{\prime}$. Using the previous lemma, we have

$$
\left|\dot{\psi}\left(x^{\prime}\right)-\dot{\psi}(x)\right| \leqslant \sum_{j=1}^{m-1}\left|\dot{\psi}\left(z_{j+1}\right)-\dot{\psi}\left(z_{j}\right)\right| \leqslant \sum_{j=1}^{m-1}\left\|\dot{\psi}\left(z_{j+1}\right)-\dot{\psi}\left(z_{j}\right)\right\|=l(C) .
$$

After taking the infimum over all chains between $x$ and $x^{\prime}$, we obtain $\left|\dot{\psi}\left(x^{\prime}\right)-\dot{\psi}(x)\right| \leqslant$ $\left|x^{\prime}-x\right|_{\dot{X}}$

We now construct a partial inverse function of $\dot{\psi}$.

Lemma 3.5.7. There is a map $\dot{\varphi}: B\left(x^{0}, \frac{r_{0}}{3}\right) \subset \lim _{\omega} \dot{X}_{n} \rightarrow \dot{X}$ such that $\dot{\varphi}$ induces a bijection onto the ball $B\left(\dot{\varphi}\left(x^{0}\right), \frac{r_{0}}{3}\right)$, whose inverse is $\dot{\psi}$. 
Proof. Let $x=\lim _{\omega} x_{n}$ be a point of $B\left(x^{0}, \frac{r_{0}}{3}\right)$. By construction, the distance between $x_{n}^{0}$ and any vertex of $\dot{X}_{n}$ is greater than $\frac{r_{0}}{2}$. Thus applying Lemma 3.1.8, we have

$$
\left|p_{n}\left(x_{n}\right)-p_{n}\left(x_{n}^{0}\right)\right|_{X_{n}} \leqslant \frac{3 \pi \sinh r_{0}}{r_{0}}\left|x_{n}-x_{n}^{0}\right|_{\dot{X}_{n}} .
$$

It follows that $\left(\left|p_{n}\left(x_{n}\right)-p_{n}\left(x_{n}^{0}\right)\right|_{X_{n}}\right)$ is $\omega$-eb. Hence $\lim _{\omega} p_{n}\left(x_{n}\right)$ defines a point in $X$. We now distinguish two cases.

(i) If there is $i \in I$ such that $x_{n}$ belongs to $C\left(Y_{n, i_{n}}\right) \omega$-as, then $x_{n}$ can be written $x_{n}=\left(p_{n}\left(x_{n}\right), r_{n}\right) \omega$-as. Since $\left(r_{n}\right)$ is bounded, we may consider $r=\lim _{\omega} r_{n}$. We define $\dot{\varphi}(x)$ as the point $\left(\lim _{\omega} p_{n}\left(x_{n}\right), r\right)$ of $C\left(Y_{i}\right)$.

(ii) If $x_{n}$ belongs to $X_{n} \omega$-as, then we define $\dot{\varphi}(x)$ as the point $\lim _{\omega} p_{n}\left(x_{n}\right)$ of $X$. The properties of $\dot{\varphi}$ are satisfied.

Lemma 3.5.8. Let $x=\lim _{\omega} x_{n}$ and $x^{\prime}=\lim _{\omega} x_{n}^{\prime}$ be two points of $B\left(x^{0}, \frac{r_{0}}{3}\right)$ such that $\left(\left\|x_{n}^{\prime}-x_{n}\right\|\right)$ is $\omega$-eb. We have $\lim _{\omega}\left\|x_{n}^{\prime}-x_{n}\right\| \geqslant\left\|\dot{\varphi}\left(x^{\prime}\right)-\dot{\varphi}(x)\right\|$.

Proof. We distinguish two cases.

(i) If there is $i \in I$ such that $x_{n}$ and $x_{n}^{\prime}$ belong to $C\left(Y_{n, i_{n}}\right) \omega$-as, then $\left\|x_{n}^{\prime}-x_{n}\right\|=$ $\left|x_{n}^{\prime}-x_{n}\right|_{C\left(Y_{n, i_{n}}\right)} \omega$-as. By continuity we have

$$
\lim _{\omega}\left|x_{n}^{\prime}-x_{n}\right|_{C\left(Y_{n, i_{n}}\right)}=\left|\dot{\varphi}\left(x^{\prime}\right)-\dot{\varphi}(x)\right|_{C\left(Y_{i}\right)} \geqslant\left\|\dot{\varphi}\left(x^{\prime}\right)-\dot{\varphi}(x)\right\| .
$$

Thus $\lim _{\omega}\left\|x_{n}^{\prime}-x_{n}\right\| \geqslant\left\|\dot{\varphi}\left(x^{\prime}\right)-\dot{\varphi}(x)\right\|$.

(ii) If $x_{n}, x_{n}^{\prime} \in X_{n} \omega$-as, but there is no $i \in I$ such that $x_{n}$ and $x_{n}^{\prime}$ belongs to $C\left(Y_{n, i_{n}}\right) \omega$-as, then $\left\|x_{n}^{\prime}-x_{n}\right\|=\left|x_{n}^{\prime}-x_{n}\right|_{X_{n}} \omega$-as. In this case we have

$$
\lim _{\omega}\left|x_{n}^{\prime}-x_{n}\right|_{X_{n}}=\left|\dot{\varphi}\left(x^{\prime}\right)-\dot{\varphi}(x)\right|_{X} \geqslant\left\|\dot{\varphi}\left(x^{\prime}\right)-\dot{\varphi}(x)\right\| .
$$

Thus $\lim _{\omega}\left\|x_{n}^{\prime}-x_{n}\right\| \geqslant\left\|\dot{\varphi}\left(x^{\prime}\right)-\dot{\varphi}(x)\right\|$.

The proof of the next corollary uses the uniform approximation of the distance on the cone-off. Indeed, if $x=\lim _{\omega} x_{n}$ and $x^{\prime}=\lim _{\omega} x_{n}^{\prime}$ are two points of $B\left(x^{0}, \frac{r_{0}}{9}\right)$, we can find for each $n$ a chain $C_{n}$ of $\dot{X}_{n}$ that approximates $\left|x_{n}^{\prime}-x_{n}\right|$ with a given error. However it is difficult to give a sense to the $\omega$-limit of $C_{n}$ if the number of points of $C_{n}$ is not uniformly bounded. That is why we previously proved Proposition 3.2.1.

Corollary 3.5.9. The restriction of $\dot{\varphi}$ to the ball $B\left(x^{0}, \frac{r_{0}}{9}\right)$ is 1-Lipschitz.

Proof. Consider two points $x=\lim _{\omega} x_{n}$ and $x^{\prime}=\lim _{\omega} x_{n}^{\prime}$ of $B\left(x^{0}, \frac{r_{0}}{9}\right)$. Let $\varepsilon>0$ such that $\left|x^{\prime}-x\right|+\varepsilon<\frac{r_{0}}{9}$. By Proposition 3.2.1, there is a number $m$ depending only of $r_{0}$ and $\varepsilon$ such that for all $n \in \mathbb{N}$ there is a chain $C_{n}=\left(z_{n}^{1}, \ldots, z_{n}^{m}\right)$ between $x_{n}$ and $x_{n}^{\prime}$ with $l\left(C_{n}\right) \leqslant\left|x_{n}^{\prime}-x_{n}\right|_{\dot{X}_{n}}+\varepsilon<\frac{r_{0}}{9}$. Notice that for all $1 \leqslant j \leqslant m$, 
$\left|z_{n}^{j}-x_{n}^{0}\right|_{\dot{X}_{n}} \leqslant\left|x_{n}-x_{n}^{0}\right|_{\dot{X}_{n}}+l\left(C_{n}\right)<\frac{r_{0}}{3}$. Thus we can consider the points $z^{j}=$ $\lim _{\omega} z_{n}^{j}$. The previous lemma gives

$\left|\dot{\varphi}\left(x^{\prime}\right)-\dot{\varphi}(x)\right|_{\dot{X}} \leqslant \sum_{j=1}^{m-1}\left\|\dot{\varphi}\left(z^{j+1}\right)-\dot{\varphi}\left(z^{j}\right)\right\| \leqslant \lim _{\omega} l\left(C_{n}\right) \leqslant \lim _{\omega}\left|x_{n}^{\prime}-x_{n}\right|_{\dot{X}_{n}}+\varepsilon$.

Hence we have $\left|\dot{\varphi}\left(x^{\prime}\right)-\dot{\varphi}(x)\right|_{\dot{X}} \leqslant\left|x^{\prime}-x\right|+\varepsilon$ for all $\varepsilon>0$. Thus $\dot{\varphi}$ is 1-Lipschitz.

Corollary 3.5.10. The map $\dot{\varphi}$ induces an isometry from the ball $B\left(x^{0}, \frac{r_{0}}{9}\right)$ onto its image.

Proof. We already know that $\dot{\varphi}$ is a 1-Lipschitz bijection from $B\left(x^{0}, \frac{r_{0}}{9}\right)$ onto its image. However its inverse function $\dot{\psi}$ is also 1-Lipschitz. Hence $\dot{\varphi}$ preserves the distances.

End of the proof of the theorem. We have just proved that $B\left(x^{0}, \frac{r_{0}}{9}\right)$ is isometric to a subset of $\dot{X}\left(Y, r_{0}\right)$. Since $\dot{X}\left(Y, r_{0}\right)$ is $\ln 3$-hyperbolic, so is $B\left(x^{0}, \frac{r_{0}}{9}\right)=$ $\lim _{\omega} B\left(x_{n}^{0}, \frac{r_{0}}{9}\right)$. Consequently there exists $n \in \mathbb{N}$ such that $B\left(x_{n}^{0}, \frac{r_{0}}{9}\right)$ is $(\ln 3+\varepsilon)$ hyperbolic. Contradiction.

3.6. Length structure on the cone-off. In order to apply the Cartan-Hadamard Theorem, we need a length structure on the cone-off. But the metric $|\cdot|_{\dot{X}}$ is not necessary a length metric. We study here the difference between $|\cdot|_{\dot{X}}$ and the length metric $d_{\dot{X}}$ induced by $|\cdot|_{\dot{X}}$. We will see that $d_{\dot{X}}$ hardly changes the geometry of $\dot{X}$. Thus if $\left(\dot{X},|\cdot|_{\dot{X}}\right)$ is hyperbolic, then so is $\left(\dot{X}, d_{\dot{X}}\right)$.

From now on, $X$ is a geodesic, $\delta$-hyperbolic space, and $Y=\left(Y_{i}\right)_{i \in I}$ a collection of strongly quasi-convex subsets of $X$. We recall that a strongly quasi-convex set $Y_{i}$ satisfies the following property: for all $x, x^{\prime} \in Y_{i}$ there exist $y, y^{\prime} \in Y_{i}$ such that the path $[x, y] \cup\left[y, y^{\prime}\right] \cup\left[y^{\prime}, x^{\prime}\right] \subset Y_{i}$ and $|y-x|,\left|y^{\prime}-x^{\prime}\right| \leqslant 10 \delta$. In particular this condition is satisfied if $Y_{i}$ is a cylinder (see Proposition 1.2.8). $\dot{X}(Y)$ is the cone-off constructed as in Section 3.1.

Lemma 3.6.1. Let $i \in I$. We have $d_{\dot{X}}\left(x, x^{\prime}\right) \leqslant\left|x^{\prime}-x\right|_{C\left(Y_{i}\right)}+40 \delta$ for all $x, x^{\prime} \in$ $C\left(Y_{i}\right)$.

Proof. We denote by $x=(y, r)$ and $x^{\prime}=\left(y^{\prime}, r^{\prime}\right)$ two points of the cone $C\left(Y_{i}\right)$. We have assumed that $Y_{i}$ was strongly quasi-convex, thus we can find two points $z$ and $z^{\prime}$ in $Y_{i}$ such that the geodesics $[y, z],\left[z, z^{\prime}\right]$ and $\left[z^{\prime}, y^{\prime}\right]$ are contained in $Y_{i}$ and $|z-y|_{X},\left|z^{\prime}-y^{\prime}\right|_{X} \leqslant 10 \delta$.

By Proposition 2.1.3, we can find a geodesic $c_{0}$ (resp. $c, c^{\prime}$ ) between $(z, r)$ and $\left(z^{\prime}, r^{\prime}\right)$ (resp. between $(y, r)$ and $(z, r)$, between $\left(y^{\prime}, r^{\prime}\right)$ and $\left.\left(z^{\prime}, r^{\prime}\right)\right)$. Since $|z-y|_{X},\left|z^{\prime}-y^{\prime}\right|_{X} \leqslant 10 \delta$, we have

$$
|(z, r)-(y, r)|_{C\left(Y_{i}\right)},\left|\left(z^{\prime}, r^{\prime}\right)-\left(y^{\prime}, r^{\prime}\right)\right|_{C\left(Y_{i}\right)} \leqslant 10 \delta .
$$


It follows that $\left|\left(z^{\prime}, r^{\prime}\right)-(z, r)\right|_{C\left(Y_{i}\right)} \leqslant\left|x^{\prime}-x\right|_{C\left(Y_{i}\right)}+20 \delta$. Thus by composing the geodesics $c, c_{0}$ and $c^{\prime}$, we obtain a path from $x$ to $x^{\prime}$ whose length is no more than $\left|x^{\prime}-x\right|_{C\left(Y_{i}\right)}+40 \delta$.

Corollary 3.6.2. For all $x, x^{\prime} \in \dot{X}$, we have $d\left(x, x^{\prime}\right) \leqslant\left\|x^{\prime}-x\right\|+40 \delta$.

Proof. Let $x$ and $x^{\prime}$ be two points of $X$. We distinguish three cases.

If there exists $i \in I$ such that $x, x^{\prime} \in C\left(Y_{i}\right)$, then $\left\|x^{\prime}-x\right\|=\left|x^{\prime}-x\right|_{C\left(Y_{i}\right)}$. Thus the inequality is given by the previous lemma.

If $x, x^{\prime} \in X$, but there is no $i \in I$ such that $x, x^{\prime} \in C\left(Y_{i}\right)$, then $\left\|x^{\prime}-x\right\|=$ $\left|x^{\prime}-x\right|_{X}$. There is a geodesic of $X$ between $x$ and $x^{\prime}$. It gives a path in $\dot{X}$, whose length is no more than $\left|x^{\prime}-x\right|_{X}$. It follows that $d(x, y) \leqslant\left\|x^{\prime}-x\right\|$.

In all the other cases, $\left\|x^{\prime}-x\right\|=+\infty$. There is nothing to prove.

Proposition 3.6.3. Let $A \geqslant 1$ and $\eta>0$. There exists a constant $\delta>0$ depending only on $A$ and $\eta$ with the following property. Let $X$ be a geodesic, $\delta$-hyperbolic space, and $Y=\left(Y_{i}\right)_{i \in I}$ a collection of strongly quasi-convex subsets of $X$. The identity map from $(\dot{X},|\cdot|)$ onto $(\dot{X}, d)$ induces a $(1, \eta)$-quasi-isometry on any ball of radius $A$.

Proof. Let $x$ and $x^{\prime}$ be two points of $\dot{X}$ such that $\left|x^{\prime}-x\right|_{\dot{X}} \leqslant 2 A$. Using Proposition 3.2.1 there exists a constant $M(A)$, an integer $m \leqslant \frac{M(A)}{\sqrt{\delta}}$ and a chain $C=$ $\left(z_{1}, \ldots, z_{m}\right)$ between $x$ and $x^{\prime}$ such that $l(C) \leqslant\left|x^{\prime}-x\right|_{\dot{X}}+\delta$. By Corollary 3.6.2 we have

$$
d\left(x, x^{\prime}\right) \leqslant \sum_{j=1}^{m-1} d\left(z_{j}, z_{j+1}\right) \leqslant l(C)+40 m \delta \leqslant\left|x^{\prime}-x\right|_{\dot{X}}+\delta+40 M(A) \sqrt{\delta} .
$$

Thus we have

$$
\left|x^{\prime}-x\right|_{\dot{X}} \leqslant d\left(x, x^{\prime}\right) \leqslant\left|x^{\prime}-x\right|_{\dot{X}}+\delta+40 M(A) \sqrt{\delta} .
$$

Consequently, if $\delta$ is small enough, the identity map from $(\dot{X},|\cdot|)$ onto $(\dot{X}, d)$ induces a $(1, \eta)$-quasi-isometry on any ball of radius $A$.

Remark. This last proposition combined with Proposition 1.0.3 tells us that if $(\dot{X},|\cdot|)$ is locally hyperbolic, then so is $(\dot{X}, d)$.

\section{Small cancellation theory}

4.1. Orbifold. In this section we introduce vocabulary concerning orbifolds. For more details about these objects see [5], Part III. $\mathscr{E}$. 


\section{Definition and length structure}

Definition 4.1.1 (Rigidity). The action of a group $G$ on a topological space $X$ is rigid if it satisfies the following property: if there is an open $U \subset X$ such that $\left.g\right|_{U}=\mathrm{id}_{U}$, then $g=1$ for all $g \in G$.

Definition 4.1.2 (Orbifold). Let $Q$ be a topological space. We say that $Q$ is an orbifold if there exists a collection $\left(U_{i}, \varphi_{i}\right)_{i \in I}$, where $U_{i}$ is a topological space and $\varphi_{i}$ a continuous map from $U_{i}$ into $Q$, satisfying the following properties.

(i) $Q=\bigcup_{i \in I} \varphi_{i}\left(U_{i}\right)$.

(ii) For all $y \in \varphi_{i}\left(U_{i}\right)$, for all $x \in \varphi_{i}^{-1}(\{y\})$, there exists a finite, rigid group of homeomorphisms of $U_{i}, G_{x}$, fixing $x$, such that $\varphi_{i} \circ g=\varphi_{i}$ for all $g \in G_{x}$ and the restriction of $\varphi_{i}$ to a neighbourhood $V_{x}$ of $x$ induces a homeomorphism from $V_{x} / G_{x}$ onto its image.

(iii) For all $x_{i} \in U_{i}$ and $x_{j} \in U_{j}$ such that $\varphi_{i}\left(x_{i}\right)=\varphi_{j}\left(x_{j}\right)$, there exists a homeomorphism $\theta_{j, i}$ from a neighbourhood of $x_{i}$ onto a neighbourhood of $x_{j}$ with $\varphi_{i}=\varphi_{j} \circ \theta_{j, i}$.

(iv) $\varphi_{i}$ lifts paths and homotopies for all $i \in I$; that is, if $c:[0,1] \rightarrow Q$ (resp. $H:[0,1] \times[0,1] \rightarrow Q)$ is a continuous path (resp. a homotopy), there exists a subdivision $0=t_{0}<\cdots<t_{p}=1$ of $[0,1]$ (resp. subdivisions $0=t_{0}<$ $\cdots<t_{p}=1$ and $0=u_{0}<\cdots<u_{q}=1$ of $\left.[0,1]\right)$ such that $\left.c\right|_{\left[t_{r}, t_{r+1}\right]}$ (resp. $\left.H\right|_{\left[t_{r}, t_{r+1}\right] \times\left[u_{s}, u_{s+1}\right]}$ ) lifts in one of the $U_{i}$.

$\left(U_{i}, \varphi_{i}\right)$ is called a chart of $Q$. The set of charts is an atlas. The map $\theta_{j, i}$ is a transition map, and the group $G_{x}$ is an isotropy group.

Definition 4.1.3 (Length structure). The orbifold defined as above is endowed with a length structure if

(i) the spaces $U_{i}$ are endowed with a length structure,

(ii) for all $x \in U_{i}$, the isotropy group $G_{x}$ is an isometry group for the length structure in $U_{i}$,

(iii) the transition maps $\theta_{j, i}$ are isometries with respect to the length structures in $U_{i}$ and $U_{j}$.

In this case, we can measure the length of a path by measuring the length of its lift.

Definition 4.1.4 ( $\sigma$-useful length structure). Let $\sigma$ be a positive number. The length structure defined as above is said to be $\sigma$-useful if for all $y \in Q$ there exists a chart $\left(U_{i}, \varphi_{i}\right)$ and a point $x \in \varphi_{i}^{-1}(\{y\})$ such that

(i) the restriction $\varphi_{i}: B(x, \sigma) \rightarrow B(y, \sigma)$ is onto,

(ii) this restriction lifts the paths starting in $y$ whose lengths are less than $\frac{\sigma}{2}$, 
(iii) this restriction lifts the homotopies $H:[0,1] \times[0,1] \rightarrow Q$ satisfying $H(0,0)=$ $y$, and for all $t_{0} \in[0,1]$ (resp. $u_{0} \in[0,1]$ ) the length of the path $u \rightarrow H\left(t_{0}, u\right)$ (resp. $\left.t \rightarrow H\left(t, u_{0}\right)\right)$ is shorter than $\frac{\sigma}{2}$.

$\left(U_{i}, \varphi_{i}, x\right)$ is called a $\sigma$-useful chart.

Definition 4.1.5 ( $\sigma$-locally $\delta$-hyperbolic length structure). Let $\sigma>0$ and $\delta>0$. The $\sigma$-useful length structure, defined as above, is said to be $\sigma$-locally $\delta$-hyperbolic if for all $y \in Q$ there exists a $\sigma$-useful chart $\left(U_{i}, \varphi_{i}, x\right)$ such that the ball $B(x, \sigma)$ is $\delta$-hyperbolic.

Topology of orbifolds. If $Q$ is an orbifold, we can define the $\mathcal{E}$-paths and the homotopy of two $\mathscr{E}$-paths (cf. [5] or [8]). This leads to the definition of the fundamental group of the orbifold $Q$ denoted by $\pi_{1}^{\text {orb }}(Q)$. We may also define the notion of covering and universal covering of $Q$ in the sense of orbifolds (cf. [5]).

Example. Let $X$ be a geodesic space and $G$ a group whose action on $X$ is rigid and proper. We denote by $Q$ the quotient $X / G$, and by $q: X \rightarrow Q$ the canonical projection. $Q$ may be endowed with an orbifold structure with one chart $(X, q)$. Indeed, for all $x \in X$, the isotropy group $G_{x}$ is necessarily finite. Moreover $q$ induces a local isometry from $X / G_{x}$ onto its image. If $X$ is simply connected, the $\operatorname{map} q: X \rightarrow Q$ is also the universal cover of $Q$ and $G=\pi_{1}^{\text {orb }}(Q)$. Such an orbifold is said to be developable.

\section{Cartan-Hadamard Theorem}

Theorem 4.1.6 ([8], Th. 4.3.1). Let $\delta>0$ and $\sigma>10^{5} \delta$. Consider an orbifold $Q$ with a $\sigma$-locally $\delta$-hyperbolic length structure. Then $Q$ is developable and its universal cover $X$ is $200 \delta$-hyperbolic. Let $(U, \varphi, x)$ be a $\sigma$-useful chart. If $z$ is a preimage in $X$ of the point $y=\varphi(x)$, then the developing map $(U, x) \rightarrow(X, z)$ induces an isometry from $B\left(x, \frac{\sigma}{10}\right)$ onto its image.

\subsection{Statement of the very small cancellation theorem}

Notation. If $G$ is a group acting on a space $X$ and $Y$ a subset of $X$, we denote by $\operatorname{Stab}(Y)$ the subgroup of $G$ that preserves $Y$, i.e.,

$$
\operatorname{Stab}(Y)=\{g \in G \mid g Y=Y\} .
$$

We define the notion of rotation family introduced by M. Gromov in [15].

Definition 4.2.1 (Rotation family). Let $\left(H_{i}\right)_{i \in I}$ be a family of subgroups of $G$ and $\left(Y_{i}\right)_{i \in I}$ a collection of pairwise distinct subspaces of $X$. We say that $\left(Y_{i}, H_{i}\right)_{i \in I}$ is a rotation family if

(i) $H_{i}$ is a finite index normal subgroup of $\operatorname{Stab}\left(Y_{i}\right)$ for all $i \in I$, 
(ii) there is an action of $G$ on $I$ which is compatible with the one on $X$, that is, we have $Y_{g i}=g Y_{i}$ and $H_{g i}=g H_{i} g^{-1}$ for all $g \in G$ and $i \in I$.

Theorem 4.2.2 (Very small cancellation theorem). There exist two positive numbers $\delta_{0}$ and $\Delta_{0}$ satisfying the following property.

Let $X$ be a geodesic, simply connected, $\delta$-hyperbolic space and $G$ a group acting properly by isometries on $X$. Let $\left(Y_{i}, H_{i}\right)_{i \in I}$ be a rotation family such that each $Y_{i}$ is strongly-quasi-convex. Let $\rho=\min _{i \in I} r_{\mathrm{inj}}\left(H_{i}, X\right), N$ be the normal subgroup of $G$ generated by the $H_{i}$ 's and $\bar{G}$ the quotient group $G / N$. Assume also that

$$
\frac{\delta}{\rho} \leqslant \delta_{0} \quad \text { and } \quad \frac{\Delta(Y)}{\rho} \leqslant \Delta_{0} .
$$

Then there exists a simply connected, hyperbolic, metric space $\bar{X}$ such that $\bar{G}$ acts properly by isometries on $\bar{X}$.

Moreover if $G$ (resp. $H_{i}$ ) acts co-compactly on $X$ (resp. $\left.Y_{i}\right)$ and $I / G$ is finite, then $\bar{X} / \bar{G}$ is compact. In particular $\bar{G}$ is hyperbolic.

Remark. In this theorem, $\Delta(Y)$ and $\rho$ respectively play the role of the length of the largest piece and the length of the smallest relation in the usual small cancellation theory.

It is important to notice that the constants $\delta_{0}$ and $\Delta_{0}$ are independent from the space $X$. This is useful in order to construct by iteration a sequence of hyperbolic groups, as it is done in [15], [8] or [1].

\subsection{Proof of the very small cancellation theorem}

Construction of an orbifold. First, we have to fix several constants in order to apply the Cartan-Hadamard Theorem 4.1.6 and the two hyperbolicity theorems (Theorems 2.3.2 and 3.5.2).

Let us consider a positive number $\varepsilon$ and choose a radius $r_{0}$ such that $r_{0}>$ $10^{6}(\ln 3+\varepsilon)$. With such constants we can apply the Cartan-Hadamard Theorem to a $\frac{r_{0}}{10}$-locally $(\ln 3+\varepsilon)$-hyperbolic orbifold. By Proposition 1.0.3, there exists a positive number $\eta$ with the following property. Consider two metric spaces $X$ and $X^{\prime}$ and a $(1, \eta)$-quasi-isometry $f: X \rightarrow X^{\prime}$. If $X^{\prime}$ is $\left(\ln 3+\frac{\varepsilon}{2}\right)$-hyperbolic then $X$ is $(\ln 3+\varepsilon)$-hyperbolic. From now on we will work with the rescaled metric space $X_{\rho}=\frac{2 \pi \sinh r_{0}}{\rho} X$. Thus, $r_{\mathrm{inj}}\left(H_{i}, X_{\rho}\right) \geqslant 2 \pi \sinh r_{0}$ for all $i \in I$.

We can find $\delta_{0}, \Delta_{0}>0$ depending on $r_{0}$ and $\varepsilon$ only such that if $\frac{\delta}{\rho} \leqslant \delta_{0}$ and $\frac{\Delta(Y)}{\rho} \leqslant \Delta_{0}$, then we have the following.

(i) Assume that $x_{0}$ is a point of $\dot{X}_{\rho}(Y)$, whose distance to a vertex is at least $\frac{r_{0}}{2}$. Then the ball $B\left(x_{0}, \frac{r_{0}}{9}\right)$ of $\left(\dot{X}_{\rho},|\cdot|_{\dot{X}_{\rho}}\right)$ is $\left(\ln 3+\frac{\varepsilon}{2}\right)$-hyperbolic (see Theorem 3.5.2).

(ii) For all $i \in I$ the cone $C\left(Y_{i}\right) / H_{i}$ is $\left(\ln 3+\frac{\varepsilon}{2}\right)$-hyperbolic (see Theorem 2.3.2). 
(iii) The identity map from $\left(\dot{X}_{\rho}, d\right)$ onto $\left(\dot{X}_{\rho},|\cdot|\right)$ restricted to any ball of radius $r_{0}$ is a $(1, \eta)$-quasi-isometry (see Proposition 3.6.3).

Thus if $x_{0}$ is a point of $\dot{X}_{\rho}(Y)$ whose distance to a vertex is at least $\frac{r_{0}}{2}$, the ball $B\left(x_{0}, \frac{r_{0}}{10}\right)$ of $\left(\dot{X}_{\rho}, d\right)$ is $(\ln 3+\varepsilon)$-hyperbolic and, for all $i \in I$, the cones $C\left(Y_{i}\right) / H_{i}$ with the length metric are $(\ln 3+\varepsilon)$-hyperbolic.

Lemma 4.3.1. The action of $G$ on $X_{\rho}$ extends to an action by isometries of $G$ on $\dot{X}_{\rho}$.

Proof. We define this action such that its restriction to $X_{\rho}$ is the action of $G$ on $X$. Let $i \in I$. Consider a point $x=(y, r)$ of $C\left(Y_{i}\right)$ and an element $g$ of $G$. We define $g \cdot x$ as the point $(g y, r)$ of $C\left(Y_{g . i}\right)$. We check that for all $x, x^{\prime} \in \dot{X}_{\rho}$ and for all $g \in G$ we have $\left\|g x^{\prime}-g x\right\|=\left\|x^{\prime}-x\right\|$. It follows that the action of $G$ preserves the distances $|\cdot|_{\dot{X}_{\rho}}$ and $d$.

From now on we denote by $Q$ the quotient space $\dot{X}_{\rho}(Y) / G$ endowed with the quotient topology. The canonical projection $\dot{X}_{\rho} \rightarrow Q$ is denoted by $q$. Then we define two kind of charts.

The first one is $(U, q)$, where $U$ is the cone-off $\dot{X}_{\rho}(Y)$ from which we have removed the vertices.

Let $i \in I$, we define $U_{i}=\left(C\left(Y_{i}\right) \backslash \iota_{i}\left(Y_{i}\right)\right) / H_{i}$. The composition $C\left(Y_{i}\right) \rightarrow$ $\dot{X}_{\rho} \rightarrow Q$ induces an map $q_{i}: U_{i} \rightarrow Q$. The second type of charts is $\left(U_{i}, q_{i}\right)$.

Lemma 4.3.2. The charts defined previously endow $Q$ with an orbifold structure.

Proof. The action of $G$ on $U$ is proper. Moreover the stabilizer of the vertex $v_{i}$ of the cone $U_{i}$ is exactly the finite group $\operatorname{Stab}\left(Y_{i}\right) / H_{i}$. We check that the atlas $\{(U, q)$, $\left.\left(U_{i}, q_{i}\right)\right\}$ defines a structure of orbifold on $Q$.

\section{Properties of the orbifold $Q$}

Lemma 4.3.3. The structure of orbifold on $Q$ defined as above is $\frac{r_{0}}{10}$-locally $(\ln 3+\varepsilon)$ hyperbolic.

Proof. It is a consequence of the two hyperbolicity theorems: the constants $\delta_{0}$ and $\Delta_{0}$ have been chosen in such a way that the structure of orbifold is $\frac{r_{0}}{10}$-locally $(\ln 3+\varepsilon)$ hyperbolic.

Corollary 4.3.4. The orbifold $Q$ is developable and its universal cover $\bar{X}$ is $\bar{\delta}$ hyperbolic, with $\bar{\delta}=200(\ln 3+\varepsilon)$.

Proof. This is an application of the Cartan-Hadamard Theorem (see Theorem 4.1.6) to the orbifold $Q$ with its $\frac{r_{0}}{10}$-locally $(\ln 3+\varepsilon)$-hyperbolic length structure. 
Proposition 4.3.5. The group $\bar{G}$ acts properly by isometries on $\bar{X}$.

Proof. We prove that $\bar{G}=\pi_{1}^{\text {orb }}(Q)$. The charts $U$ and $U_{i}$ are simply connected. This implies that $\pi_{1}^{\text {orb }}(U / G)=G$ and $\pi_{1}^{\text {orb }}\left(U_{i} /\left(\operatorname{Stab}\left(Y_{i}\right) / H_{i}\right)\right)=\operatorname{Stab}\left(Y_{i}\right) / H_{i}$. Applying to $Q$ the van Kampen Theorem for orbifolds, it follows that $\pi_{1}^{\text {orb }}(Q)=$ $\operatorname{Stab}\left(Y_{i}\right) / H_{i} * \operatorname{Stab}\left(Y_{i}\right) G=\bar{G}$ (see [16]). Thus $\bar{G}$ acts properly on $\bar{X}$.

Proposition 4.3.6. If $G$ (resp. $H_{i}$ ) acts co-compactly on $X$ (resp. $Y_{i}$ ) and $I / G$ is finite, then $\bar{X} / \bar{G}$ is compact. In particular $\bar{G}$ is hyperbolic.

Proof. Since there are, up to a translation by an element of $G$, a finite number of $Y_{i}$, it follows that $Q$ is obtained by gluing a finite number of compact cones on the compact space $X / G$. Thus $Q=\bar{X} / \bar{G}$ is compact. Hence the action of $\bar{G}$ on $\bar{X}$ is proper, co-compact.

Theorem 4.3.7. Let $X$ be an $n$-dimensional simplicial complex with $8(n+1) \bar{\delta} \leqslant \frac{r_{0}}{100}$. Suppose that, for all $x \in X$ and all $r \in \mathbb{R}^{+}$, there exists a homotopy $h: \bar{B}(x, r) \times$ $[0,1] \rightarrow X$ contracting $\bar{B}(x, r)$ to $\{x\}$ such that $\left|h\left(x^{\prime}, t\right)-x\right| \leqslant\left|x^{\prime}-x\right|+\frac{\bar{\delta} \rho}{2 \pi \sinh r_{0}}$ for all $x^{\prime} \in \bar{B}(x, r)$. Moreover, suppose that the $Y_{i}$ 's have the same property. Then $\bar{X}$ is contractible.

Proof. Let $\bar{x}$ be a point of $\bar{X}$ and $r \in[0,8(n+1) \bar{\delta}]$. We denote by $\bar{B}$ the closed ball $\bar{B}(\bar{x}, r)$ of $\bar{X}$. We distinguish two cases.

Case 1 . There exists $i \in I$ such that the vertex $\bar{v}_{i}$ of the cone $C\left(Y_{i}\right) / H_{i}$ belongs to $\bar{B}$. Then there is a homotopy $H: \bar{B} \times[0,1] \rightarrow \bar{B}$ which contracts $\bar{B}$ to $\left\{\bar{v}_{i}\right\}$.

Case 2. The ball $\bar{B}$ does not contain a vertex $\bar{v}_{i}$. Due to the Cartan-Hadamard Theorem, there exists a chart $(V, x)$ such that the developing map $(V, x) \rightarrow(\bar{X}, \bar{x})$ induces an isometry from $B\left(x, \frac{r_{0}}{100}\right)$ onto its image. In particular the ball $\bar{B}$ lifts in one of the charts. Assume that this chart is $U_{i}=C\left(Y_{i}\right) / H_{i}$. Since $\bar{v}_{i}$ does not belong to $\bar{B}$, the ball $\bar{B}$ lifts in fact in the cone $C\left(Y_{i}\right)$. By Proposition 2.4.1, $\bar{B}$ is contractible in $B(\bar{x}, r+\bar{\delta})$. In the other hand, if the chart $V$ is $U$, we apply Proposition 3.3.1. Thus $\bar{B}$ is contractible in $B(\bar{x}, r+3 \bar{\delta})$.

Consequently, every ball $\bar{B}(\bar{x}, r)$ of $\bar{X}$, with $r \leqslant 8(n+1) \bar{\delta}$, is contractible in $B(\bar{x}, r+4 \delta)$. By Proposition 1.3.4, $\bar{X}$ is contractible.

\section{Examples of aspherical complexes}

In this section we explain how to construct examples of rotation families satisfying the very small cancellation assumptions. Let $X$ be a proper, geodesic, $\delta$-hyperbolic space and $Y$ a closed convex subset of $X$. Let $G$ be a group acting properly, co-compactly, by isometries on $X$, such that $\operatorname{Stab}(Y)$ acts co-compactly on $Y$. 
We are interested in the rotation family $\left(g Y, g H^{-1}\right)_{g \in G / \operatorname{Stab}(Y)}$, where $H$ is a subgroup of $\operatorname{Stab}(Y)$. In concrete situations, $\left\{\operatorname{diam}\left(g Y^{+20 \delta} \cap Y^{+20 \delta}\right) \mid g \in\right.$ $G \backslash \operatorname{Stab}(Y)\}$ may not be bounded. Nevertheless, in many situations this assumption can be achieved by replacing $G$ with a finite index subgroup of $G$. This uses, as explained in the next lemma, the profinite topology of groups.

Lemma 5.1. Assume that the subgroup $\operatorname{Stab}(Y)$ is closed in $G$ under the profinite topology. Let $\Delta$ be a non-negative number. There exists a finite index subgroup $G^{\prime}$ of $G$ containing $\operatorname{Stab}(Y)$ such that for all $g$ in $G^{\prime}$ we have $g \in \operatorname{Stab}(Y)$ if and only if $\operatorname{diam}\left(g Y^{+20 \delta} \cap Y^{+20 \delta}\right)>\Delta$.

Proof. Let $K$ be a compact fundamental domain for the action of $\operatorname{Stab}(Y)$ on $Y$. Since the action of $G$ is proper, the set

$$
E=\left\{g \in G \mid \operatorname{diam}\left(g K^{+\Delta+30 \delta} \cap K^{+\Delta+30 \delta}\right)>\Delta\right\}
$$

is finite. By assumption, $\operatorname{Stab}(Y)$ is closed in $G$ under the profinite topology. In other words,

$$
\operatorname{Stab}(Y)=\bigcap_{\substack{N \triangleleft G \\[G: N]<+\infty}} \operatorname{Stab}(Y) \cdot N .
$$

Hence there exists a finite index normal subgroup $N$ of $G$ such that $E \cap \operatorname{Stab}(Y) \cdot N \subset$ $\operatorname{Stab}(Y)$. We denote by $G^{\prime}$ the $\operatorname{set} \operatorname{Stab}(Y) \cdot N$. It is a finite index subgroup of $G$ containing $\operatorname{Stab}(Y)$. Consider now $g \in G^{\prime}$ such that $\operatorname{diam}\left(g Y^{+208} \cap Y^{+20 \delta}\right)>\Delta$. Since $K$ is a fundamental domain of $Y$, there exist four points $x, x^{\prime}, y, y^{\prime}$ of $K^{+308+\Delta}$ and two elements $h, h^{\prime}$ of $\operatorname{Stab}(Y)$ with the following properties: $h x=g h^{\prime} x^{\prime}$, $h y=g h^{\prime} y^{\prime}$ and $|y-x|=\left|y^{\prime}-x^{\prime}\right|>\Delta$. Thus $h^{-1} g h^{\prime}$ belongs to $E \cap G^{\prime}$. But $h$ and $h^{\prime}$ both belong to $\operatorname{Stab}(Y)$. Hence $g \in \operatorname{Stab}(Y)$.

In this context, the following result of N. Bergeron is useful.

Proposition 5.2 ([2], Lemme principal). Let $\Lambda$ be an algebraic subgroup of $\mathrm{GL}_{n}(\mathbb{R})$ and $G$ a finitely generated subgroup of $\mathrm{GL}_{n}(\mathbb{R})$. Then $\Lambda \cap G$ is closed in $G$ under the profinite topology.

The second lemma explains how to find a subgroup $H$ of $\operatorname{Stab}(Y)$ with an injectivity radius as large as desired.

Lemma 5.3. Assume that the group $G$ is residually finite. Then for all $\rho>0$ there exists a finite index normal subgroup $H$ of $\operatorname{Stab}(Y)$ such that $r_{\mathrm{inj}}(H, X) \geqslant \rho$.

Proof. Let $K$ be a compact fundamental domain for the action of $G$ on $X$. Since the action of $G$ is proper, the set

$$
E=\left\{g \in G \mid g K^{+\rho} \cap K^{+\rho} \neq \emptyset\right\}
$$


is finite. Moreover, $G$ is residually finite. Hence there exists a finite index normal subgroup $N$ of $G$ such that $E \cap N=\{1\}$. Consider $g \in N \backslash\{1\}$ and $x \in X$. By definition there exists $h \in G$ such that $h x \in K$. But $h g h^{-1}$ belongs to $N \backslash\{1\}$, thus $h g h^{-1} K^{+\rho} \cap K^{+\rho}=\emptyset$. It follows that $|g x-x|=\left|\left(h g h^{-1}\right) h x-h x\right| \geqslant \rho$. Hence $[g] \geqslant \rho$. Consequently, we take for $H$ the group $N \cap \operatorname{Stab}(Y)$.

Theorem 5.4. Let $\mathbb{H}_{n}$ denote the real (resp. complex, quaternionic) hyperbolic space, and $\delta$ its hyperbolicity constant. We consider $\Lambda_{k}=\mathrm{SO}(k, 1)$ (resp. $\mathrm{SU}(k, 1)$, $\mathrm{Sp}(k, 1))$ as the stabilizer of $\mathbb{H}_{k}$ in $\mathbb{H}_{n}$. Let $G$ be a uniform lattice of $\Lambda_{n}=\mathrm{SO}(n, 1)$ (resp. $\mathrm{SU}(n, 1), \mathrm{Sp}(n, 1))$. We assume that $G \cap \Lambda_{k}$ is a uniform lattice of $\Lambda_{k}$.

(i) There exists a finite index subgroup $G^{\prime}$ of $G$ such that the set

$$
\left\{\operatorname{diam}\left(g \mathbb{H}_{k}^{+20 \delta} \cap \mathbb{H}_{k}^{+20 \delta}\right) \mid g \in G^{\prime} \backslash \Lambda_{k}\right\}
$$

is bounded.

(ii) Let $\bar{Q}$ be the space obtained by gluing a cone of base $\mathbb{H}_{k} / G^{\prime} \cap \Lambda_{k}$ over $\mathbb{H}_{n} / G^{\prime}$. There is a finite index subgroup $H$ of $G^{\prime} \cap \Lambda_{k}$ and a contractible hyperbolic space $\bar{X}$ such that $\bar{G}^{\prime}=G^{\prime} /\left\langle\langle H\rangle\right.$ acts properly co-compactly on $\bar{X}$, and $\bar{Q}=\bar{X} / \bar{G}^{\prime}$.

Proof. Applying Proposition 5.2, $G \cap \Lambda_{k}$ is closed in $G$ under the profinite topology. The first point follows from Lemma 5.1.

We denote by $\Delta$ the upper bound of

$$
\left\{\operatorname{diam}\left(g \mathbb{H}_{k}^{+20 \delta} \cap \mathbb{H}_{k}^{+20 \delta}\right) \mid g \in G^{\prime} \backslash \Lambda_{k}\right\} .
$$

It is known that a finitely generated subgroup of $\Lambda_{n}$ is residually finite. Using Lemma 5.3 there exists a finite index normal subgroup $H$ of $G^{\prime} \cap \Lambda_{k}$, whose injectivity radius is $\rho$, and such that $\delta \leqslant \delta_{0} \rho$ and $\Delta \leqslant \Delta_{0} \rho$, where $\delta_{0}$ and $\Delta_{0}$ are the constants given by the very small cancellation theorem (see Theorem 4.2.2). It follows that the rotation family $\left(g \mathbb{H}_{k}, g H g^{-1}\right)_{g \in G^{\prime} / G^{\prime} \cap \Lambda_{k}}$ satisfies the hypotheses of the very small cancellation theorem. Thus there exists a hyperbolic space $\bar{X}$ such that $\bar{G}^{\prime}=$ $\left.G^{\prime} /\langle H\rangle\right\rangle$ acts properly by isometries on $\bar{X}$ and $\bar{Q}=\bar{X} / \bar{G}^{\prime}$. Since $G^{\prime}$ (resp. $H$ ) is a uniform lattice of $\Lambda_{n}$ (resp. $\Lambda_{k}$ ) the action of $G^{\prime}$ (resp. $H$ ) on $\mathbb{H}_{n}$ (resp. $\mathbb{H}_{k}$ ) is co-compact. It follows that the action of $\bar{G}^{\prime}$ on $\bar{X}$ is co-compact. Moreover, every ball in $\mathbb{H}_{n}$ or $\mathbb{H}_{k}$ is contractible. Thus Theorem 4.3.7 implies that $\bar{X}$ is contractible.

The next result is proved in the same way, it only uses another kind of convex subsets of $\mathbb{H}_{n}$.

Theorem 5.5. Let $\mathbb{H}_{n}(\mathbb{C})$ denote the complex hyperbolic space, and let $\delta$ be its hyperbolicity constant. Consider $\mathrm{SO}(n, 1)$ as the stabilizer of the $n$-dimensional real hyperbolic space $\mathbb{H}_{n}(\mathbb{R})$ in $\mathbb{H}_{n}(\mathbb{C})$. Let $G$ be a uniform lattice of $\mathrm{SU}(n, 1)$ such that $G \cap \mathrm{SO}(n, 1)$ is also a uniform lattice of $\mathrm{SO}(n, 1)$. 
(i) There exists a finite index subgroup $G^{\prime}$ of $G$ such that the set

$$
\left\{\operatorname{diam}\left(g \mathbb{H}_{n}(\mathbb{R})^{+20 \delta} \cap \mathbb{H}_{n}(\mathbb{R})^{+20 \delta}\right) \mid g \in G^{\prime} \backslash \mathrm{SO}(n, 1)\right\}
$$

is bounded.

(ii) Denote by $\bar{Q}$ the space obtained by attaching over $\mathbb{H}_{n} / G^{\prime}$ a cone of base $\mathbb{H}_{n}(\mathbb{R}) / G^{\prime} \cap \mathrm{SO}(n, 1)$. There is a finite index subgroup $H$ of $G^{\prime} \cap \mathrm{SO}(n, 1)$ and a contractible hyperbolic space $\bar{X}$ such that $\bar{G}^{\prime}=G^{\prime} /\langle\langle H\rangle\rangle$ acts properly co-compactly on $\bar{X}$, and $\bar{Q}=\bar{X} / \bar{G}^{\prime}$.

\section{References}

[1] G. N. Arzhantseva and T. Delzant, Examples of random groups. Preprint 2008. http://www.unige.ch/math/folks/arjantse/Abs/random.pdf

[2] N. Bergeron, Premier nombre de Betti et spectre du laplacien de certaines variétés hyperboliques. Enseign. Math. (2) 46 (2000), 109-137. Zbl 0969.58009 MR 1769939

[3] N. Bergeron, Lefschetz properties for arithmetic real and complex hyperbolic manifolds. Internat. Math. Res. Notices 2003 (2003), 1089-1122. Zbl 1036.11021 MR 1963482

[4] N. Bourbaki, Éléments de mathématique. Topologie générale. Chapitres 1 à 4 . Hermann, Paris 1971. Zbl 0249.54001 MR 0358652

[5] M. R. Bridson and A. Haefliger, Metric spaces of non-positive curvature. Grundlehren Math. Wiss. 319, Springer-Verlag, Berlin 1999. Zbl 0988.53001 MR 1744486

[6] M. Coornaert, T. Delzant, and A. Papadopoulos, Géométrie et théorie des groupes. Lecture Notes in Math. 1441, Springer-Verlag, Berlin 1990. Zbl 0727.20018 MR 1075994

[7] R. Coulon, Automorphismes extérieurs du groupe de Burnside libre. PhD Dissertation, Université de Strasbourg, Strasbourg 2010.

http://tel.archives-ouvertes.fr/tel-00489295/fr/

[8] T. Delzant and M. Gromov, Courbure mésoscopique et théorie de la toute petite simplification. J. Topol. 1 (2008), 804-836. Zbl 1197.20035 MR 2461856

[9] C. Druţu, Cônes asymptotiques et invariants de quasi-isométrie pour des espaces métriques hyperboliques. Ann. Inst. Fourier (Grenoble) 51 (2001), 81-97. Zbl 0992.20031 MR 1821069

[10] C. Druţu, Quasi-isometry invariants and asymptotic cones. Internat. J. Algebra Comput. 12 (2002), 99-135. Zbl 1010.20029 MR 1902363

[11] C. Druţu and M. Sapir, Tree-graded spaces and asymptotic cones of groups. Topology 44 (2005), 959-1058. Zbl 1101.20025 MR 2153979

[12] É. Ghys and P. de la Harpe (eds.), Sur les groupes hyperboliques d'après Mikhael Gromov. Progr. Math. 83, Birkhäuser, Boston 1990. Zbl 0731.20025 MR 1086648

[13] M. Gromov, Hyperbolic groups. In Essays in group theory, Math. Sci. Res. Inst. Publ. 8, Springer-Verlag, New York 1987, 75-263. Zbl 0634.20015 MR 0919829 
[14] M. Gromov, Mesoscopic curvature and hyperbolicity. In Global differential geometry: the mathematical legacy of Alfred Gray (Bilbao, 2000), Contemp. Math. 288, Amer. Math. Soc., Providence, RI, 2001, 58-69. Zbl 1006.53036 MR 1871000

[15] M. Gromov, Random walk in random groups. Geom. Funct. Anal. 13 (2003), 73-146. Zbl 1122.20021 MR 1978492

[16] A. Haefliger and Q. N. Du, Appendice: une présentation du groupe fondamental d'une orbifold. Astérisque (1984), 98-107. Zbl 0556.57032 MR 755164

[17] R. C. Lyndon and P. E. Schupp, Combinatorial group theory. Ergeb. Math. Grenzgeb. 89, Springer-Verlag, Berlin 1977. Zbl 0368.20023 MR 0577064

[18] Y. Ollivier, On a small cancellation theorem of Gromov. Bull. Belg. Math. Soc. Simon Stevin 13 (2006), 75-89. Zbl 1129.20022 MR 2245980

[19] A. Yu. Ol'shanskii, Geometry of defining relations in groups. Math Appl. (Soviet Series) 70, Kluwer Academic Publishers, Dordrecht 1991. Zbl 0732.20019 MR 1191619

[20] P. Papasoglu, On the asymptotic cone of groups satisfying a quadratic isoperimetric inequality. J. Differential Geom. 44 (1996), 789-806. Zbl 0893.20029 MR 1438192

[21] J. H. C. Whitehead, On adding relations to homotopy groups. Ann. of Math. (2) 42 (1941), 409-428. JFM 67.0738.04 Zbl 0027.26404 MR 0004123

[22] J. H. C. Whitehead, Note on a previous paper entitled "On adding relations to homotopy groups”. Ann. of Math. (2) 47 (1946), 806-810. Zbl 0060.41104 MR 0017537

[23] D. T. Wise, Cubulating small cancellation groups. Geom. Funct. Anal. 14 (2004), 150-214. Zbl 1071.20038 MR 2053602

Received August 25, 2009; revised February 8, 2010

R. Coulon, Vanderbilt University, Department of Mathematics, 1326 Stevenson Center, Nashville, TN 37240, U.S.A.

E-mail: remi.coulon@vanderbilt.edu 